\title{
The brightest stars of the $\sigma$ Orionis cluster
}

\author{
J. A. Caballero`
}

\author{
Max-Planck-Institut für Astronomie, Königstuhl 17, 69117 Heidelberg, Germany \\ e-mail: caballero@mpia.de \\ Isaac Newton Group, Apartado 321, 38700 Santa Cruz de La Palma, Canary Islands, Spain \\ Instituto de Astrofísica de Canarias, 38205 La Laguna, Tenerife, Spain
}

Received 27 October 2006 / Accepted 2 January 2007

\begin{abstract}
Context. The very young $\sigma$ Orionis cluster ( $\sim 3 \mathrm{Ma}$ ) is a cornerstone in understanding the formation of stars and substellar objects down to planetary masses. However, its stellar population is far from being completely known.

Aims. This study's purpose is to identify and characterise the most massive stars of $\sigma$ Orionis to complement current and future deep searches for brown dwarfs and planetary-mass objects in the cluster.

Methods. I have cross-correlated the sources in the Tycho and 2MASS catalogues in a region of 30 arcmin radius with its centre in the O-type star $\sigma$ Ori A. In this area, I studied the membership in the Ori OB 1b association of the brightest stars in the optical using astrometric, X-ray, and both infrared and optical photometric data from public catalogues, and spectroscopic data from the literature. Results. A list of 26 young stars, four candidate young stars, and 16 probable foreground stars has arisen from the study. Seven young stars probably harbour discs (four are new). There is no mass dependence of the disc frequency in the cluster. I have derived the first mass spectrum for $\sigma$ Orionis from 1.1 to $24 M_{\odot}\left(\alpha=+2.0_{-0.1}^{+0.2}\right.$; roughly Salpeter-like). I also provide additional proof of the existence of several spatially superimposed stellar populations in the direction of $\sigma$ Orionis. Finally, the cluster may be closer and older than previously thought $^{\star \star}$.
\end{abstract}

Key words. open clusters and associations: individual: $\sigma$ Orionis - stars: general - planetary systems: protoplanetary discs astronomical data bases: miscellaneous

\section{Introduction}

The constellation of Orion, the Hunter, is dominated by the stellar components of the Ori OB 1 complex. This complex is probably related to the Barnard loop, a huge feature with $\mathrm{H} \alpha$ in emission that extends up to Eridanus, and to a hole in the distribution of gas $\mathrm{H} \mathrm{I}$, which may have originated in several supernova events that happened about $4 \mathrm{Ma}$ ago (Brown et al. 1994). The very young Orion complex is a set of four subgroups or associations of very early type stars, named Ass Ori OB 1-a, b, $\mathrm{c}$, and $\mathrm{d}$. Each of them possesses a slightly different location, age $(1-10 \mathrm{Ma})$, and heliocentric distance $(300-500 \mathrm{pc})$. Blaauw (1964), Warren \& Hesser (1978), Goudis (1982), de Geus et al. (1990), Brown et al. (1994), and other authors have described the boundaries between subgroups and their characteristics.

The $\sigma$ Orionis cluster lies within the Ori OB 1b association, also known as the Orion Belt. This association contains three famous perfectly aligned, very bright stars: Alnitak ( $\zeta$ Ori), Alnilam ( $\epsilon$ Ori) and Mintaka ( $\delta$ Ori). It has been suggested that the age, distance and radial velocity of the stellar components vary across the association (Hardie et al. 1964; Warren \& Hesser 1977a,b; Guetter 1981; Gieseking 1983; Genzel \& Stutzki 1989). The eastern part of the Ori OB 1b association,

^ Alexander von Humboldt Fellow at the Max-Planck-Institut für Astronomie.

$\star \star$ Tables A.1 and A.2 are also available in electronic form at the CDS via anonymous ftp to

cdsarc.u-strasbg.fr $(130.79 .128 .5)$ or via

http://cdsweb.u-strasbg.fr/cgi-bin/qcat?J/A+A/466/917 with Alnitak, the Horsehead Nebula, the Flame Nebula (the Orion B cloud, associated to NGC 2024) and the H II region IC 434, would be the farthest and youngest one. Actually, the star formation is still underway close to the Horsehead Nebula, whose mane is illuminated by the $\sigma$ Ori star ${ }^{1}$, the fourth brightest star in the Orion Belt.

Garrison (1967) and Lyngå (1981) were the first investigators to recognise the $\sigma$ Orionis cluster. It went unnoticed until Wolk (1996) and Walter et al. (1998) detected an over-density of X-ray sources and a population of pre-main-sequence low-mass stars surrounding the centre of the cluster (see also: Walter et al. 1997; Wolk \& Walter 2000). Although it is not as extremely young as the Trapezium cluster in the Ori OB 1d association (the Orion Sword) and is not as near to the Sun as the rich star-forming regions in the southern hemisphere (i.e. Chamaeleon, Ophiuchus), the $\sigma$ Orionis cluster has the advantage of simultaneously possessing a moderate youth, a relatively short heliocentric distance, and especially, a very low interstellar extinction (Lee et al. 1968; Béjar et al. 2004b). This fact may be due to strong winds, ultraviolet radiation and/or turbulence generated by the O-type star in the cluster centre, which would sweep away the gas and dust. Furthermore, several authors have suggested that $\sigma$ Orionis is in reality an H II region (e.g. Reipurth et al. 1998).

There are different ways to determinate the age of the $\sigma$ Orionis cluster based on different methodologies: $(i)$ from the comparison between mid-resolution real and theoretical spectra

\footnotetext{
1 Through this paper and for clarity, I am using the full and shortened names for stars and clusters, respectively; i.e. the $\sigma$ Ori star system, the $\sigma$ Orionis cluster.
} 
Table 1. Different determinations of the age of the $\sigma$ Orionis cluster.

\begin{tabular}{lr}
\hline \hline Age (Ma) & Reference \\
\hline 8 & Blaauw (1964) \\
$<5.1^{a}$ & Warren \& Hesser (1978) \\
7 & Blaauw (1991) \\
$1.7 \pm 1.1$ & Brown et al. (1994) \\
$4.2^{+2.7}$ & Oliveira et al. (2002) \\
$2-4^{b}$ & Zapatero Osorio et al. (2002b) \\
$2.5 \pm 0.3$ & Sherry et al. (2004) \\
$3.5 \pm 3.0$ & Hernández et al. (2005) \\
$4-6$ & Briceño et al. (2005) \\
6.6 & Kharchenko et al. (2005) \\
$\lesssim 4-6^{c}$ & Sacco et al. (2006) \\
$4-5$ & Mayne et al. (2006) \\
\hline
\end{tabular}

${ }^{a}$ Age not rotation-corrected (upper limit: 5.1 Ma).

${ }^{b}$ Upper limit: $8 \mathrm{Ma}$; lower limit: $1 \mathrm{Ma}$.

${ }^{c}$ They also found three low-mass high probability members that are older than $\sim 10 \mathrm{Ma}$.

surrounding the Li I $\lambda 6707.8 \AA$ youth-indicator line; (ii) from the presence of very early-type stars; (iii) from the disc frequency around stars; and (iv) from fits to theoretical isochrones in colour-magnitude diagrams. All these determinations are directed towards a narrow age interval between 0.5 and $8 \mathrm{Ma}$. In Table 1, I summarise several age determinations found in the literature. The most probable age of the cluster is about $3 \mathrm{Ma}$, with conservative -2 and +5 Ma error-bars.

The heliocentric distance to $\sigma$ Ori from the trigonometric parallax measured by the Hipparcos mission is $352_{-168}^{+166} \mathrm{pc}$. There are also different determinations of the heliocentric distance to the Ori OB $1 \mathrm{~b}$ association and to several $\sigma$ Orionis members (Brown et al. 1994, 1999; Perryman et al. 1997; de Zeeuw et al. 1999; Brown et al. in prep.). Published distances vary between $360_{-60}^{+70} \mathrm{pc}$ and $473 \pm 33 \mathrm{pc}$. The most recent determinations of the heliocentric distance to the $\sigma$ Orionis cluster were shown by Hernández et al. (2005), who determined the distances from parallax, $443 \pm 16 \mathrm{pc}$, and from colour-magnitude diagrams, $392 \pm 20 \mathrm{pc}$, and by Sherry et al. (2004), who determined a heliocentric distance of $440 \pm 40 \mathrm{pc}$. I used the value measured by Brown et al. (1994) of $360_{-60}^{+70} \mathrm{pc}$.

Béjar et al. (1999) opened the era of deep photometric searches in the cluster and detected the first substellar objects in $\sigma$ Orionis, which do not have stable hydrogen burning in their cores, in contrast to stars. Later studies have gone deeper, discovering a rich population of brown dwarfs (Béjar et al. 2001, 2004b; Scholz \& Eislöffel 2004; Caballero et al. 2004; Kenyon et al. 2005) and even objects with masses in the planetary domain (below the deuterium-burning mass limit) (Zapatero Osorio et al. 2000; González-García et al. 2006; Caballero et al. 2007a). The $\sigma$ Orionis cluster is to date the star-forming region with the largest amount of candidate planetary-mass objects (27). Out of them, 12 have low-resolution spectroscopy (Martín et al. 2001; Barrado y Navascués et al. 2001), and three harbour surrounding discs (Caballero et al. 2007b). The cluster has now turned out to be not only the region with the highest stellar density in the Ori OB $1 \mathrm{~b}$ association, but one of the most interesting regions in the Milky Way for studying and understanding the formation of stars, brown dwarfs, and especially, isolated planetary-mass objects.

In spite of the importance of the cluster, its stellar population is paradoxically poorly known. General studies of the Ori OB 1b association (e.g. Warren \& Hesser 1977a,b, 1978), extensive searches with prism-objective and Schmidt plates
(Haro \& Moreno 1953; Kogure et al. 1989; Wiramihardja et al. 1989, 1991; Nakano et al. 1995) and the ROSAT-based search by Wolk (1996) provided most of the known cluster members at the end of last century. However, these surveys were obviously biased towards the detection of active Ori OB1 1b stellar members. Some efforts have been recently carried out to improve our knowledge of the stellar population in $\sigma$ Orionis. Nonetheless, the bias towards $\mathrm{H} \alpha$ emitters (Weaver \& Babcock 2004), largeamplitude photometric variables (Scholz \& Eislöffel 2004), or low-mass objects (Tej et al. 2002; Sherry et al. 2004) is still prevalent. The summit of our relatively poor knowledge of the stellar and high-mass brown-dwarf population in the cluster is maybe the case of SE 70. Scholz \& Eislöffel (2004) detected it at 4.6 arcsec from a previously known $\sim 5 M_{\text {Jup }}$-mass candidate planetary-mass object, S Ori 68 (Zapatero Osorio et al. 2000). Caballero et al. (2006a) have recently confirmed the membership in the cluster of SE 70, which is an X-ray-emitter brown dwarf $^{2}$ and may form, together with S Ori 68, the widest planetary system ever found $(1700 \pm 300 \mathrm{AU})$. The recent discovery of at least two spatially coincident stellar populations in the area makes current and future studies more complex. The superimposed stellar components, first shown by Jeffries et al. (2006) and confirmed by Sacco et al. (2006), are kinematically separated in radial velocity and have different mean ages. Furthermore, $\sigma$ Orionis itself might not be a member of any of the known Ori OB1 subgroups, but instead part of a new one. For example, the simple fact of the Horsehead Nebula in the Ori OB $1 \mathrm{~b}$ association being a back-illuminated absorption nebula may suggest that it is at a different heliocentric distance than $\sigma$ Orionis.

A complete study of the stellar population in the $\sigma$ Orionis cluster is absolutely needed to complement current and future deep searches of brown dwarfs and planetary-mass objects in the area (e.g. to compare spatial distributions, disc frequencies, or slopes of the mass spectrum at different mass intervals and separations from nearby massive stars). In this paper, I describe an optical and near-infrared search for the most massive stars in the area of $\sigma$ Orionis, supported by astrometric, X-ray, and mid-infrared measurements. The search and characterisation of intermediate- and low-mass stars in the cluster will be presented in a forthcoming paper.

\section{Analysis}

A cross match between the Tycho- 2 and 2MASS catalogues will lead to the identification of the brightest stars (in the optical) of the Ori OB $1 \mathrm{~b}$ association in the direction of the $\sigma$ Orionis cluster. Collecting additional data from different archives related to mid-infrared and X-ray space missions and from the literature will provide information on the nature and age of the stars.

\subsection{The Tycho-2/2Mass correlation}

For the present work, I have used the web-based sky atlas Aladin (v3.600; Bonnarel et al. 2000; Fernique et al. 2004). I loaded all the sources catalogued in the Tycho-2 and the 2MASS Point Source catalogues (Høg et al. 2000 and Cutri et al. 2003, respectively) in a circle of $30 \mathrm{arcmin}$ radius centred in $\sigma$ Orionis $\mathrm{A}+\mathrm{B}+\mathrm{IRS} 1$ (the close triple OB system formed by $\sigma$ Ori A, $\sigma$ Ori B and $\sigma$ Ori IRS1 that gives the name to the cluster).

2 Burningham et al. (2005) also measured the equivalent width of the Na I $\lambda \lambda 8183,8195 \AA$ doublet and the radial velocity of SE 70, which are very similar to those of other confirmed young brown dwarfs in the $\sigma$ Orionis cluster. 


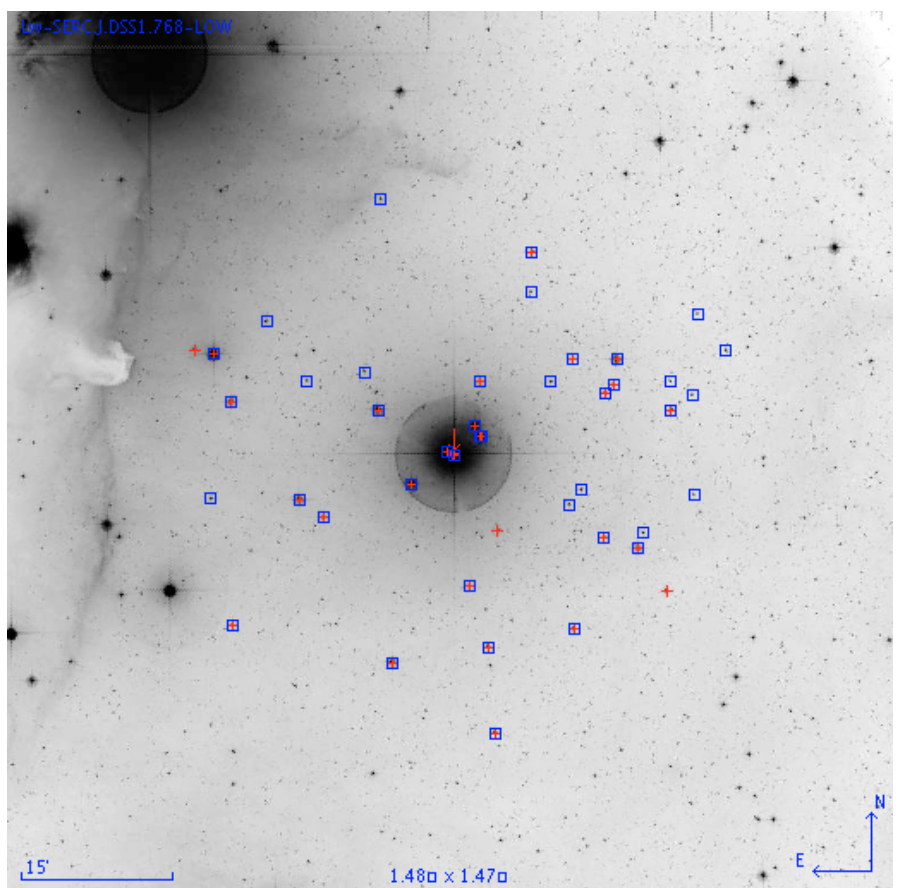

Fig. 1. Inverse-colour DSS-1 blue-band (photographic $B_{J}$ ) image centred in $\sigma$ Ori A (marked with an arrow). Its size is $1.5 \times 1.5 \mathrm{deg}^{2}$. North is up and East is left. Sources in Tycho-1 and Tycho-2 are marked with (red) crosses and (blue) squares, respectively (in colour in the electronic version). East and northeast of the field of view are dominated by the star-forming region complex formed by Alnitak, the Flame Nebula (NGC 2024), the Horsehead Nebula and the IC $434 \mathrm{H}$ II region.

In this area of $\sim 2830 \mathrm{arcmin}^{2}$, there are 41 Tycho- 2 and about 5700 2MASS catalogued sources. Previous studies in the region point out that $30 \operatorname{arcmin}(\sim 3 \mathrm{pc}$ at the distance of $\sigma$ Orionis) is a rough but valid approximation of the cluster radius (Sherry et al. 2004; Béjar et al. 2004a). Many bright stars out of this radius may be related to the youngest star-forming regions to the east of the Ori OB $1 \mathrm{~b}$ association or to the Alnilam cluster to the west (see e.g. Wolk 1996). The B1.5V-type star HD 37744 and the Horsehead Nebula are in the vicinity of the studied region.

The positional cross match in the area with the Aladin cross match tool between the Tycho- 2 and 2MASS catalogues provided a list of 41 stellar sources. Therefore, the 2MASS nearinfrared counterparts of all the Tycho- 2 sources in the area were identified. I used the default cross-match threshold of 4 arcsec. Except in one case, the agreement between the Tycho- 2 and 2MASS coordinates was better than 0.5 arcsec (and better than 0.2 arcsec in $78 \%$ of the cases). The difference between the Tycho- 2 and 2MASS coordinates of the outlier was 2.4 arcsec. This source (TYC 47701432 1), with a magnitude in the reddest Tycho band $V_{\mathrm{T}}=12.3 \pm 0.2 \mathrm{mag}$, is one of the faintest stars in the sample. The deviation may be ascribed to the low signal-tonoise ratio in the Tycho catalogue, where the $90 \%$-completeness magnitude in the $V$ band is about $11.5 \mathrm{mag}$ (Høg et al. 2000).

Figure 1 shows a $\sim 1.5 \times 1.5 \mathrm{deg}^{2}$ blue digitized photographic plate, covering the region of 30 arcmin centred in $\sigma$ Ori A and adjacent areas. The 41 correlated stars are marked in the view window with squares. The photometry in five bands $\left(B_{\mathrm{T}} V_{\mathrm{T}} J H K_{\mathrm{s}}\right)$ and accurate proper motion measurements for each star is available. In the upper parts of Tables A.1 and A.2, I provide their names and the photometric and astrometric data, respectively. The stars in the Tycho-1 catalogue (ESA 1997), superseded in most applications by Tycho-2, are marked with
Table 2. Mid-infrared sources among the stars in the Tycho-2/2Mass correlation.

\begin{tabular}{lcccc}
\hline \hline Name & $F_{12}$ & $F_{25}$ & $F_{60}$ & $F_{100}$ \\
& {$[\mathrm{Jy}]$} & {$[\mathrm{Jy}]$} & {$[\mathrm{Jy}]$} & {$[\mathrm{Jy}]$} \\
\hline$\sigma$ Ori A+B+IRS1 $^{a}$ & $4.5 \pm 0.2$ & $15 \pm 2$ & $15 \pm 2$ & $15 \pm 2$ \\
HD 37699 & $0.29:$ & $0.45 \pm 0.09$ & $1.5 \pm 0.2$ & $4.9:$ \\
HD 294268 & $0.87 \pm 0.11$ & $2.74 \pm 0.16$ & $1.50 \pm 0.10$ & $22:$ \\
\hline
\end{tabular}

${ }^{a}$ Re-measured IRAS photometry (Oliveira \& van Loon 2004).

crosses in Fig. 1. There are four additional stars that are in Tycho-1 and not in Tycho-2.

\subsection{Additional data}

\subsubsection{IRAS space telescope}

Three out of the 41 correlated stars possess an unambiguous source in the IRAS catalogue of Point Sources Version 2.0 (IPAC 1986) at less than 40 arcsec. Their names and average noncolour corrected flux densities, $F_{v}$, for $12,25,60$, and $100 \mu \mathrm{m}$ are given in Table 2 . These three stars have been investigated as mid-infrared sources with probable discs $(\sigma$ Ori A+B+IRS1 - van Loon \& Oliveira 2004; HD 294268 - García-Lario et al. 1990, 1997 and Torres et al. 1995; HD 37699 - Oganesyan et al. 1995).

In the studied area there are only four additional IRAS unambiguous sources associated to fainter stars and their surrounding envelopes. They are TX Ori, V510 Ori, IRAS 05358-0238, and IRAS 05352-0227. None of them are in the Tycho-2 catalogue. However, the correlated star HD 294269 has an unreliable detection at only one passband, $60 \mu \mathrm{m}$, and will not be considered.

\subsubsection{ROSAT, XMM-Newton and Chandra space telescopes}

At least 13 stars in the sample are X-ray emitters. They were found in the following catalogues: ROSAT Source Catalog of Pointed Observations with the High Resolution Imager, 1RXH (ROSAT 2000a); WGACAT version of the ROSAT PSPC Catalogue, WGACAT (White et al. 2000); Second ROSAT Source Catalog of Pointed Observations, 2RXP (ROSAT 2000b).

All the X-ray emitters, except $\sigma$ Orionis E and the faint star 4771-0950 (Wolk 1996), are present in the WGACAT-ROSAT catalogue. Nine were previously detected in the spatial and spectral analysis of a full EPIC field in the XMM-Newton observations of the $\sigma$ Orionis cluster by Franciosini et al. (2006). They reported deviations between the coordinates of the optical and the X-ray sources of less than 1 arcsec for all the bright stars in their sample. Additionally, HD 37699 and HD 294268, to the northeast of the area, were also detected in the X-ray observations of the Orion OB $1 \mathrm{~b}$ association with the ASCA mission by Nakano et al. (1999). Voges et al. (1999) considered GSC 04771-00962 and GSC 04771-00961 as possible HST Guide Star Catalogue optical counterparts of the X-ray ROSAT source 1RXS J053756.8-023857, which is in reality ascribed to the bright young star 2E 0535.4-0241. Table 3 shows the X-ray emitters in the sample, the count rates, and the number of events measured by different instruments and space missions.

The high background produced by the extreme X-ray emitter $\sigma$ Ori $\mathrm{A}+\mathrm{B}+\mathrm{IRS} 1$ did not allow me to recognise $\sigma$ Ori $\mathrm{E}$ as an individual WGACAT source. Likewise, the small angular separation between HD 294272 A and B ( 8 arcsec) and their similarity in brightness did not permit me to discriminate 
Table 3. X-ray sources among the stars in the Tycho-2/2Mass correlation $^{a}$.

\begin{tabular}{lccc}
\hline \hline Name & $\begin{array}{c}\text { Nr. } \\
\text { 1RXH }\end{array}$ & $\begin{array}{c}\text { WGACAT } \\
{\left[10^{-3} \mathrm{~s}^{-1}\right]}\end{array}$ & $\begin{array}{c}\text { XMM } \\
{\left[10^{-3} \mathrm{~s}^{-1}\right]}\end{array}$ \\
\hline$\sigma$ Ori A+B+IRS1 & 129 & $330 \pm 80$ & $440 \pm 2$ \\
$\sigma$ Ori E & 77 & - & $199.4 \pm 1.7$ \\
HD 37699 & 4 & $90 \pm 20$ & $\times$ \\
HD 37525 AB & 129 & $19.6 \pm 1.3$ & $45.7 \pm 0.9$ \\
HD 294272 A+B & 3 & $3.6 \pm 0.6$ & $5.9 \pm 0.4$ \\
HD 37564 & 4 & $24 \pm 5$ & $9.9 \pm 0.5$ \\
V1147 Ori & $\times$ & $70 \pm 40$ & $\times$ \\
HD 294268 & $\times$ & $3.50 \pm 0.11$ & $\times$ \\
RX J0539.6-0242 AB & 142 & $60 \pm 20$ & $63 \pm 2$ \\
HD 294298 & $\times$ & $60 \pm 8$ & $\times$ \\
4771-0950 & 1 & - & $0.67 \pm 0.18$ \\
2E 0535.4-0241 & 160 & $53 \pm 17$ & $189 \pm 4$ \\
\hline
\end{tabular}

${ }^{a}$ Name of optical counterpart, number of events in 1RXH-ROSAT, count rate $\left(\mathrm{s}^{-1}\right)$ in WGACAT-ROSAT and count rate $\left(\mathrm{s}^{-1}\right)$ in the XMMNewton observations by Franciosini et al. (2006). The ROSAT and XMM-Newton missions are not sensitive to the same wavelength interval. The symbol " $X$ " denotes that the target is not in the area studied by the X-ray mission or catalogue.

which is the real X-ray emitter from ROSAT and XMM-Newton data. However, both $\sigma$ Ori $\mathrm{A}+\mathrm{B}+\mathrm{IRS} 1$ and $\sigma$ Ori $\mathrm{E}$ and the HD 294272 A and B systems are resolved in the high-spatialresolution X-ray images from the Chandra Space Telescope archive obtained by Adams et al. (2004) with HRI-C. The null detection at high energies of the rest of the targets, except in the case of StHA 50, may be due to the large separation to $\sigma$ Ori A, where X-ray surveys are usually centred.

\subsubsection{Optical spectroscopy}

From my searches in the literature, 28 stars have a spectral type determination. Their spectral types are provided in the last column of Table A.2. Out of them, 13 stars (14 if the Betype star StHa 50 is also included) have OBA spectral types. Another five stars have F types, and the rest are G- and Ktype stars. The spectral-type determinations have been borrowed from the following works: Schild \& Chaffee (1971), Warren \& Hesser (1978), Guetter (1981), Downes \& Keyes (1988), Gray \& Corbally (1993), Nesterov et al. (1995), Wolk (1996), Catalano \& Renson (1998), Alcalá et al. (2000), Gregorio-Hetem \& Hetem (2000), and Caballero (2006).

There is information about the lithium abundance for seven stars. In six cases, the equivalent width of the Li I $\lambda 6707.8 \AA$ suggests that the lithium abundance is primordial $(\log \epsilon(\mathrm{Li}) \sim$ 3.0), while there is an upper limit of 1.0 dex for the $\log \epsilon(\mathrm{Li})$ of the G0-type star HD 294269 (Cunha et al. 1995; Torres et al. 1995; Alcalá et al. 1996, 2000; Caballero 2006). See a careful study in Cunha et al. (1995) of the lithium abundance of late $\mathrm{F}$ and early $\mathrm{G}$ stars in Orion and how very young ages can be derived for stars with effective temperatures between $\sim 5200$ and $\sim 6400 \mathrm{~K}$.

There are three stars with $\mathrm{H} \alpha$ in emission: RX J0539.6-0242 AB $(E W(\mathrm{H} \alpha)=-2.38 \AA$; Alcalá et al. 1996), StHA 50 (Stephenson 1986; Downes \& Keyes 1988) and HD 294268 $(E W(\mathrm{H} \alpha)=-2 \AA$; Torres et al. 1995). Likewise, seven stars display spectroscopic peculiarities: V1147 Ori (abnormally strong silicon and europium lines; Catalano \& Renson 1998), HD 37333 (mild Si II; Gray \& Corbally 1993), HD 37699, HD 37686 and HD 294301 (broad -“nebulous"- absorption
Table 4. Very young stars in the Tycho-2/2MASS correlation.

\begin{tabular}{|c|c|c|c|c|c|c|}
\hline Name & $\mathrm{OB}$ & X-ray & Li I & $\mathrm{H} \alpha$ & mIR & var. \\
\hline$\sigma$ Ori $\mathrm{A}+\mathrm{B}+\mathrm{IRS} 1$ & $\mathrm{Y}$ & $\mathrm{Y}$ & & & $\mathrm{Y}$ & \\
\hline$\sigma$ Ori E & Y & Y & & & & $\mathrm{Y}$ \\
\hline HD 37699 & Y & Y & & & Y & \\
\hline HD 294271 & $\mathrm{Y}$ & & & & & \\
\hline HD $37525 \mathrm{AB}$ & $\mathrm{Y}$ & $\mathrm{Y}$ & & & & \\
\hline HD 294272 A & Y & $\mathrm{Y}$ & & & & \\
\hline HD 294272 B & Y & Y & & & & \\
\hline HD 37333 & Y & & & & & \\
\hline HD 37564 & & $\mathrm{Y}$ & & & & \\
\hline V1147 Ori & $\mathrm{Y}$ & Y & & & & $\mathrm{Y}$ \\
\hline HD 37686 & Y & & & & & \\
\hline HD 37545 & Y & & & & & \\
\hline HD 294275 & Y & & & & & \\
\hline HD 294268 & & $\mathrm{Y}$ & $\mathrm{Y}$ & $\mathrm{Y}$ & $\mathrm{Y}$ & Y? \\
\hline RX J0539.6-0242 AB & & Y & Y & Y & & \\
\hline HD 294279 & & & Y & & & \\
\hline StHA 50 & Y? & & & $\mathrm{Y}$ & & \\
\hline HD 294298 & & $\mathrm{Y}$ & $\mathrm{Y}$ & & & \\
\hline 4771-0950 & & $\mathrm{Y}$ & Y & & & \\
\hline 2E 0535.4-0241 & & Y & & & & \\
\hline SO120532 & & & $\mathrm{Y}$ & & & \\
\hline
\end{tabular}

due to spinning; Neubauer 1943; Warren \& Hesser 1978 and Gray \& Corbally 1993, respectively), HD 37525 AB (helium weak; Nissen 1976) and $\sigma$ Ori $\mathrm{E}$ (helium rich and magnetic strong; Greenstein \& Wallerstein 1958). The stars HD 294298 and RX J0539.6-0242 AB also have high values of $v \sin i$ if compared with field solar-like stars (55 and $150 \mathrm{~km} \mathrm{~s}^{-1}$, respectively; Cunha et al. 1995 and Alcalá et al. 1996). Finally, Hunger et al. (1989) measured a $v \sin i$ of $160 \pm 20 \mathrm{~km} \mathrm{~s}^{-1}$ in $\sigma$ Ori E.

\subsection{Target classification}

\subsubsection{Young stars}

The primordial abundance of lithium ("Li I"), the line of $\mathrm{H} \alpha$ in appreciable emission in solar-type stars or earlier ("H $\alpha$ "), the mid-infrared flux excess in the IRAS pass-bands due to a disc ("mIR"), the very early spectral types (O, B and early A; "OB"), the X-ray emission (strong if a solar-type star; "X"), the highamplitude photometric variability ("var."), and the fast rotation are some known youth features (age $\lesssim 10 \mathrm{Ma}$ ). These signatures are common in $\mathrm{T}$ Tauri stars and their analogous at intermediate masses, the Herbig $\mathrm{Ae} / \mathrm{Be}$ stars (with masses in the interval $1.5 M_{\odot}<M<10 M_{\odot}$ and spectral types earlier than F; Hillenbrand et al. 1992). Out of the 41 stars of the Tycho-2 sample, 21 have at least one youth feature. In Table 4, I provide the list of the very young stars. I consider that they are bona-fide members of the Ori OB $1 \mathrm{~b}$ association, to which the $\sigma$ Orionis cluster belongs. Some of the stars display several youthful features at the same time, such as HD 294268, which is a mid-infrared source with moderate X-ray and $\mathrm{H} \alpha$ emissions and lithium in absorption. It is also a probable photometric variable star. Warren \& Hesser (1978) suggested the membership in the Ori OB $1 \mathrm{~b}$ association of 14 stars among the subsample of young stars in Table 4.

\subsubsection{Stars with high tangential velocity}

The tabulated mean proper motion of all the subgroups of the Ori OB1 association is $\mu_{\alpha} \cos \delta=+0.44 \mathrm{mas} \mathrm{s}^{-1}, \mu_{\delta}=-0.65 \mathrm{mas} \mathrm{s}^{-1}$ (Brown et al. 1999), while de Zeeuw et al. (1999) show that 


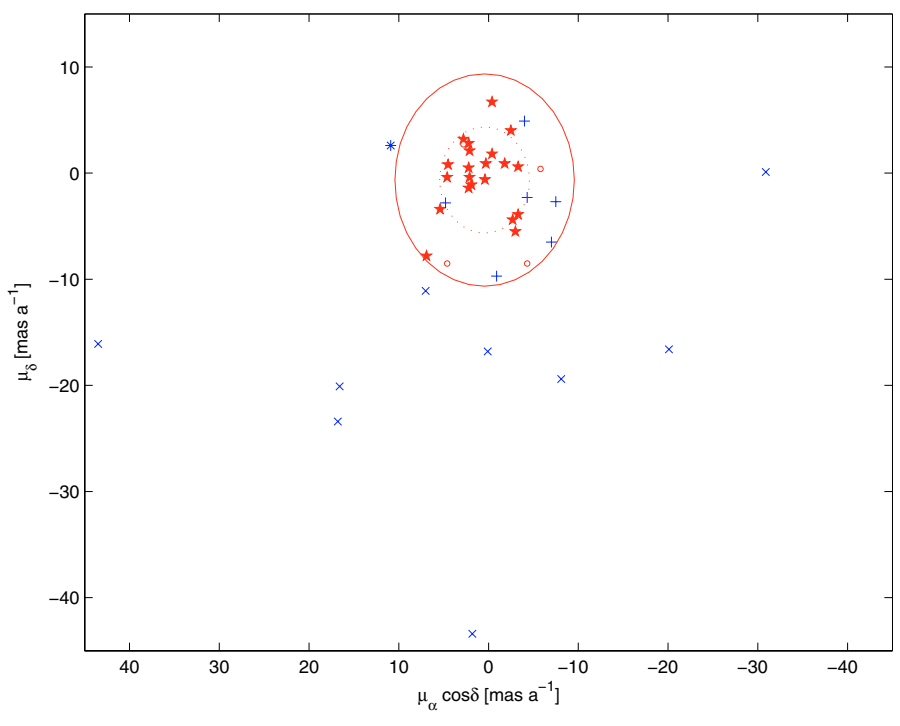

Fig. 2. Proper motions of the 41 Tycho-2/2MASS-correlation stars.

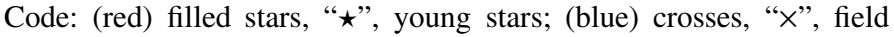
stars with proper motion different from the mean proper motion of the Ori OB 1 b association; (blue) pluses, "+”, probable K- or M-type foreground stars; (red) open circles, "o", possible new Ori OB 1b association members. The dotted and solid big circles (ellipses with the axes scale) denote the Brown et al. (1999) and this paper's astrometric selection criteria, respectively. The object marked with an asterisk, "*”, is HD 294278, which is a K-type foreground star with a different proper motion ("X" and "+" simultaneously).

the tangential velocities of $96 \%$ of the Ori OB1 photometric candidates identified by Brown et al. (1994) deviated less than 5 mas s$^{-1}$ from that mean proper motion. However, in the work presented here, there is a large fraction of young stars that display features of extreme youth, and their tangential velocity does not satisfy the de Zeeuw et al. (1999) proper-motion criterion. One extreme case is that of the F7-type X-ray-emitter star 4771-0950, with $\mathrm{p} E W(\mathrm{Li}$ I) $=+0.07 \pm 0.01 \AA$ (Caballero 2006), whose proper motion deviates in almost 10 mas s$^{-1}$ from the mean proper motion of the Ori OB1 association. I have used the value of 10 mas s$^{-1}$ to separate young stars and candidate association members from probable foreground stars with higher tangential velocities. This wider "error circle" is not caused by larger errors in the proper motions of the faintest members, but due to a genuine tangential velocity dispersion (see Sect. 3.3). Figure 2 illustrates the astrometric study, showing the selection criteria by Brown et al. (1999) and the one used here. The ten stars with tangential velocity different from that of the Ori OB1 association in more than 10 mas s$^{-1}$ are shown in Table 5 (they could be up to $3 \mathrm{deg}$ from $\sigma$ Ori $\mathrm{AB}$ in $1 \mathrm{Ma}$ ). The association membership status of HD 294270 in Warren \& Hesser (1978) was uncertain.

\subsubsection{K- and M-type foreground stars}

In the $V_{\mathrm{T}}$ vs. $V_{\mathrm{T}}-K_{\mathrm{s}}$ colour-magnitude diagram shown in Fig. 3, there are two well-differentiated regions separated at $V_{\mathrm{T}} \sim 9.5 \mathrm{mag}$. On the one hand, the 13 stars that are brighter than this value are bluer than $V_{\mathrm{T}}-K_{\mathrm{s}}=1.0$ mag display youth features and, hence, verify the astrometric criterion. On the other hand, out of the 28 stars fainter than $V_{\mathrm{T}} \sim 9.5 \mathrm{mag}$, ten are the propermotion outliers shown in Table 5. There are also seven stars, shown in Table 6 , with $V_{\mathrm{T}}-K_{\mathrm{s}}$ colours redder than $\sim 3.0 \mathrm{mag}$, which are located far from the photometric cluster sequence
Table 5. Stars in the Tycho-2/2MASS correlation with proper motion different from that of the Ori OB1 association in more than 10 mas s$^{-1}$.

\begin{tabular}{lccr}
\hline \hline Name & $\begin{array}{c}\mu_{\alpha} \cos \delta \pm \delta \mu_{\alpha} \cos \delta \\
{\left[\mathrm{mas} \mathrm{a}^{-1}\right]}\end{array}$ & $\begin{array}{c}\mu_{\delta} \pm \delta \mu_{\delta} \\
{\left[\mathrm{mas} \mathrm{a}^{-1}\right]}\end{array}$ & $\begin{array}{r}\text { Sp. } \\
\text { type }\end{array}$ \\
\hline HD 294307 & $+0.1 \pm 1.4$ & $-16.8 \pm 1.4$ & F8 \\
GSC 04771-00621 & $+7.0 \pm 1.6$ & $-11.1 \pm 1.6$ & \\
HD 294278 & $+10.9 \pm 1.4$ & $+2.6 \pm 1.4$ & K2 \\
HD 294270 & $+16.8 \pm 1.9$ & $-23.4 \pm 1.8$ & G0 \\
HD 294269 & $+43.5 \pm 1.8$ & $-16.1 \pm 1.8$ & G0 \\
HD 294274 & $-8.1 \pm 3.2$ & $-19.4 \pm 3.5$ & G0 \\
TYC 4771 720 1 & $-20.1 \pm 3.9$ & $-16.6 \pm 4.2$ & \\
TYC 4771 661 1 & $+16.6 \pm 4.3$ & $-20.1 \pm 4.7$ & \\
TYC 4770 1432 1 & $-30.9 \pm 4.5$ & $+0.1 \pm 5.0$ & \\
TYC 4770 924 1 & $+1.8 \pm 4.7$ & $-43.4 \pm 5.1$ & \\
\hline
\end{tabular}

${ }^{a}$ Non member of the Ori OB $1 \mathrm{~b}$ association in Warren \& Hesser (1978). ${ }^{b}$ No detection of Li I in absorption and $V_{r}=+72.8 \mathrm{~km} \mathrm{~s}^{-1}$ in Cunha et al. (1995).

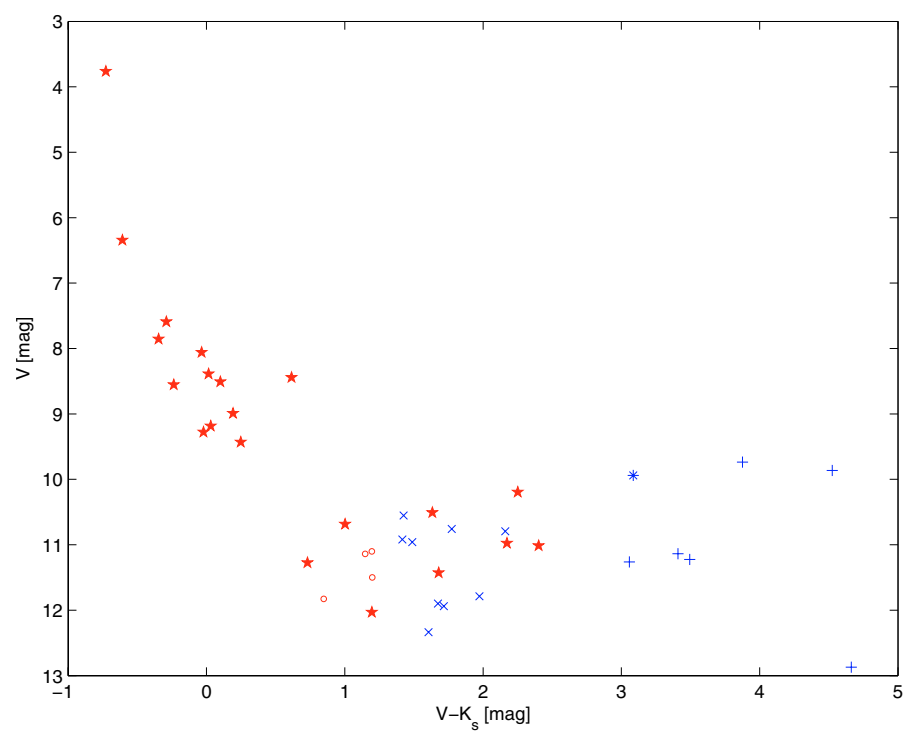

Fig. 3. Same as Fig. 2 but for the $V_{\mathrm{T}}$ vs. $V_{\mathrm{T}}-K_{\mathrm{S}}$ colour-magnitude diagram.

defined by the young stars. Their $V_{\mathrm{T}}-K_{\mathrm{S}}$ colours and $V_{\mathrm{T}}$ apparent magnitudes are typical of early/intermediate $\mathrm{K}$ dwarfs in the foreground. Furthermore, Nesterov et al. (1995) determined the $\mathrm{K}$ spectral type for the three brightest stars. Given their brightness (with $J$ magnitudes brighter than $9.5 \mathrm{mag}$ ), if they were association members, then they should be extraordinarily reddened stars showing the astrophysical properties of Class I objects. None of them shows X-ray or mid-infrared emissions detectable by present surveys, nor any spectroscopic youth feature. On the contrary, the three young members of the Ori OB $1 \mathrm{~b}$ association with $V_{\mathrm{T}}-K_{\mathrm{S}}$ colours between 2.0 and $2.5 \mathrm{mag}$ and of similar brightness in the $V_{\mathrm{T}}$ band to the stars in Table 6 are among the strongest X-ray emitters in the $\sigma$ Orionis cluster (RX J0539.6-0242 AB, 2E 0535.4-0241, and HD 294298). The K2 star HD 294278 does not satisfy the astrometric selection criterion either.

\subsubsection{Possible new Ori OB $1 \mathrm{~b}$ association members}

There are four stars that have not been classified as young stars, proper-motion outliers, or foreground K-type stars and that follow the photometric sequence of the normal young stars. They 
Table 6. Probable K- and M-type foreground stars in the Tycho2/2MASS correlation.

\begin{tabular}{lr}
\hline \hline Name & Sp. type \\
\hline HD 294277 & K2 \\
HD 294278 & K2 \\
HD 294280 & K5 \\
TYC 4770 1018 1 & \\
TYC 4771 1012 1 & \\
TYC 4771 934 1 & \\
TYC 4771 14681 & \\
\hline
\end{tabular}

a They were found by the Midcourse Space Experiment satellite at $8.3 \mu \mathrm{m}$ (Kraemer et al. 2003).

Table 7. Possible new Ori OB $1 \mathrm{~b}$ association member stars in the Tycho-2/2MASS correlation.

\begin{tabular}{lc}
\hline \hline Name & Sp. type \\
\hline HD 294301 & F2V(n) \\
GSC 04771-00962 & \\
TYC 4770 1261 1 & \\
TYC 4770 1129 1 & \\
\hline
\end{tabular}

are tabulated in Table 7. There is available spectroscopic information only for the fast-rotating F2-type star HD 294301 (Gray \& Corbally 1993), whose association membership status in Warren \& Hesser (1978) was also uncertain. The star GSC 04771-00962 might be related to an unidentified X-ray event in the Second ROSAT PSPC Catalog, at 0.7 arcmin to the north of the star. I consider the four stars as possible new Ori OB $1 \mathrm{~b}$ association members.

\subsection{Binary systems}

Among these targets, there are at least four close multiple systems. They are $\sigma$ Ori A+B+IRS1, HD 37525 AB, HD 294272 $\mathrm{A}$ and $\mathrm{B}$ and the spectroscopic binary RX J0539.6-0242 AB. Both Tycho-2 and 2MASS observations were able to resolve the HD 294272 system. It is not the case, however, for the other three stars. The secondary at $0.45 \pm 0.4 \operatorname{arcsec}$ of HD $37525 \mathrm{~A}$ is more than 0.5 mag fainter in the $H$ band than the primary, so the contribution of the $\mathrm{B}$ component to the total flux at optical wavelengths may not be appreciable. The same occurs, in an extreme case, with $\sigma$ Ori IRS1, which is more than 5 mag fainter than $\sigma$ Ori AB in the $H$ band (Caballero 2005, 2006). However, the optical flux of $\sigma$ Ori B is only three times less than that of $\sigma$ Ori A. Although the short separation between both components (of 0.250 arcsec in J1991.25), it is possible to estimate the optical magnitudes of $\sigma$ Ori A and B from Hipparcos data. The combined $H_{P}$ magnitude of $\sigma$ Ori $\mathrm{AB}$ is $3.681 \pm 0.016 \mathrm{mag}$, while the magnitude difference between them, $\Delta H_{p}$, is $1.21 \pm 0.05 \mathrm{mag}$. Using the $B-H_{P}$ and $H_{P}-V$ colours of blue Hipparcos stars as a reference, I have estimated that the Johnson $B$ - and $V$-band magnitudes of $\sigma$ Ori $\mathrm{A}$ and $\mathrm{B}$ are 3.85 and $4.10 \mathrm{mag}$ and 5.18 and $5.26 \mathrm{mag}$, respectively, with errors of $\sim 0.05 \mathrm{mag}$. I used the relationships $\Delta m_{A+B}=m_{B}-m_{A}$ and $m_{A}=m_{A+B}+2.5 \log \left(1+10^{-\frac{\Delta m_{A+B}}{2.5}}\right)$. The $V_{A}$ and $V_{B}$ estimations agree nicely with $V$-band adaptive optics observations of $\sigma$ Ori AB by ten Brummelaar et al. (2000). The spectroscopic binary RX J0539.6-0242 AB cannot be resolved by imaging with current technology. Tovmassian et al. (1991) also propose a suspected binary among the correlated stars, HD 37564, but it has not been confirmed.
Table 8. Young stars in the Tycho-1 catalogue and not in the Tycho-2 catalogue.

\begin{tabular}{lcccr}
\hline \hline Name & OB & X-ray & Li I & Sp. type \\
\hline$\sigma$ Ori D & Y & Y & & B2V \\
HD 294273 & Y & & Y & F6-8 \\
HD 294297 & & & Y \\
\hline${ }^{a} v \sin i=180 \pm 20 \mathrm{~km} \mathrm{~s}^{-1}$ (Hunger et al. 1989).
\end{tabular}

\subsection{Missing stars}

\subsubsection{Young stars in the Tycho-1 catalogue}

The Tycho-2 catalogue is not complete. As mentioned before, there are four stars in Tycho-1 that are not in Tycho-2. Their basic properties (photometry, astrometry, spectral types) are tabulated in the bottom parts of Tables A.1 and A.2. The stars are brighter than or as bright as the Tycho $V_{\mathrm{T}}$ completeness magnitude. Furthermore, one of them is $\sigma$ Ori $\mathrm{D}$, which is one of the brightest stars in the $\sigma$ Orionis cluster. The four stars roughly follow the cluster sequence in the $V_{\mathrm{T}}$ vs. $V_{\mathrm{T}}-K_{\mathrm{S}}$ colour-magnitude diagram. Their proper motion measurements are affected by effects external to the Hipparcos satellite (like the glare of $\sigma$ Ori $\mathrm{A}+\mathrm{B}+\mathrm{IRS} 1$ in the case of $\sigma$ Ori $\mathrm{D}$ or the background emission of the Horsehead Nebula in the case of HD 294297). Errors in their proper motions in Tycho- 1 are larger than $5 \mathrm{mas} \mathrm{a}^{-1}$ and up to 30 mas a $^{-1}$, and Tycho- 2 does not even tabulate them. Table A.2 shows the tangential velocities of the four stars provided by $\mathrm{H} \varnothing \mathrm{g}$ et al. (1998), which matches at the level of a few mas a ${ }^{-1}$ with the USNO-B1 and NOMAD1 catalogues (Monet et al. 2003 and Zacharias et al. 2004, respectively).

Out of the four missing stars, three display youthful features (Cunha et al. 1995; Sanz-Forcada et al. 2004; Caballero 2005, 2006). The young missing stars are shown in Table 8 . Very interestingly, HD 294297 has both a primordial lithium abundance and a proper motion that clearly deviates from the rest of the Ori OB $1 \mathrm{~b}$ association members. Its tangential velocity is $\left(\mu_{\alpha} \cos \delta, \mu_{\delta}\right)=(+22.7 \pm 2.6,-20.5 \pm 1.7) \mathrm{mas} \mathrm{a}^{-1}$ from Høg et al. $(1998)$ and $(+22.1 \pm 1.6,-23.9 \pm 1.4) \mathrm{mas} \mathrm{a}^{-1}$ from the NOMAD1 catalogue. The remaining missing target, the G0-type star HD 294276, does not display any youth feature and has the highest tangential velocity among the stars studied in this work, so it is likely a foreground solar-like star.

\subsection{2. $\sigma$ Ori $\mathrm{C}$}

The Hipparcos mission was mostly sensitive to the blue optical wavelengths. There are no stars brighter than $J=9.0 \mathrm{mag}$ in the 2MASS catalogue and in the surveyed area without an optical counterpart in the Tycho catalogues. However, there are 12 2MASS stars with magnitudes $9.0<J \leq 10.0$ mag not found in Tycho-1 or 2. Among them are four foreground Kand M-type stars (based on mid-resolution optical spectroscopy and/or proper motion; Caballero 2006), five cluster members, and three stars of an unknown nature. Out of the five young stars, the brightest one is the A2-type star $\sigma$ Ori $\mathrm{C}$, which is about 2.7 mag brighter in the $V$ band than the $90 \%$-completeness magnitude of the Tycho-2 catalogue. Its absence in the Tycho catalogues may be for the same reason as $\sigma$ Ori $\mathrm{D}$ in the optical and $\sigma$ Ori E in X rays: the glare of $\sigma$ Ori AB. I will "recover" $\sigma$ Ori $\mathrm{C}$ as one of the brightest $\sigma$ Orionis cluster members. 


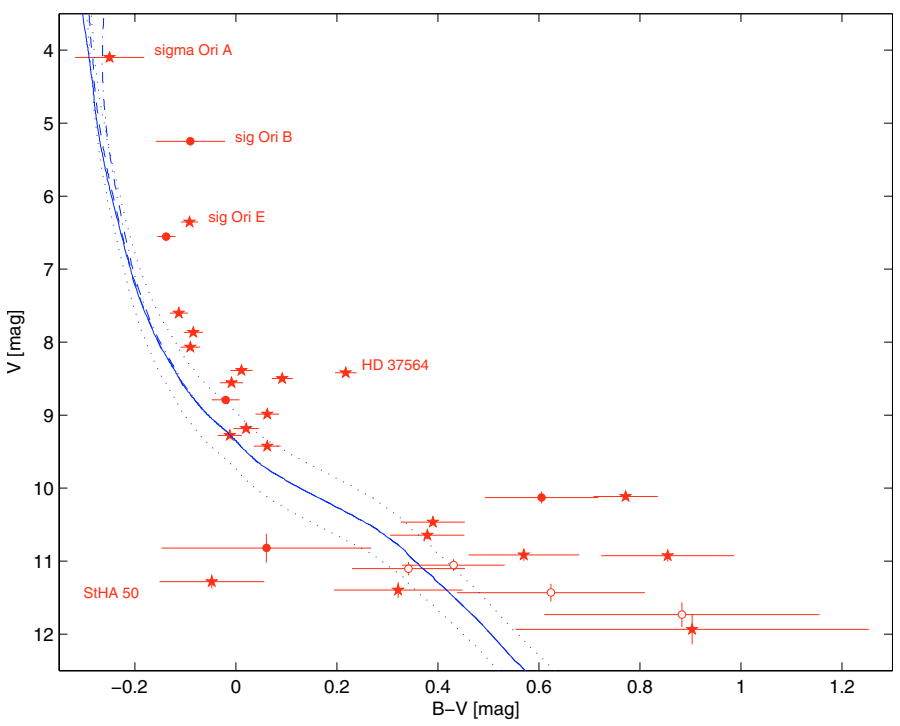

Fig. 4. $V$ vs. $B-V$ colour-magnitude diagram of the young stars in the Tycho-2/2MASS correlation (filled stars), the four new probable Ori OB $1 \mathrm{~b}$ association members (open circles), $\sigma$ Ori $\mathrm{B}, \mathrm{C}$, and the three young stars in the Tycho-1 recovery (filled circles). Solid, dashed, and dot-dashed lines (in overlapping) denote the 1-, 3-, and 10-Ma isochrones of the Geneva group at the most probable heliocentric cluster distance, and the dotted lines indicate the 3-Ma isochrones at the minimum $(300 \mathrm{pc})$ and maximum $(430 \mathrm{pc})$ possible cluster distances. Several targets are labeled.

\section{Discussion}

To sum up, out of the 41 stars in the Tycho- 2 catalogue at angular separations less than 30 arcmin of $\sigma$ Ori A, 21 stars have youth features according to my search in catalogues and in the literature. I have also computed the $B$ - and $V$-band magnitudes of $\sigma$ Ori A and B separately and considered four additional bright young stars (three from the Tycho- 1 catalogue plus $\sigma$ Ori C) and four new candidate Ori OB $1 \mathrm{~b}$ association members. This makes a list of 26 bright young stars and four candidate young stars in the studied area. Out of them, nine are in multiple systems: $\sigma$ Ori A, B, C, D, and E, HD $294272 \mathrm{~A}$ and B, HD $37525 \mathrm{AB}$, and RX J0539.6-0242 AB. It indicates that the minimum frequency of multiplicity of the brightest stars is $35 \%$ (the low-mass stars and substellar objects are not taken into account). Next, I characterise the sample of young stars.

\subsection{Individual masses and the $V$ vs. $B-V$ diagram}

For each of the 30 bright young stars and candidates, I derived their masses from the Johnson $B$ - and $V$-band and theoretical stellar evolution isochrones from the Geneva database (Lejeune \& Schaerer 2001). Johnson magnitudes were derived from Tycho $B_{\mathrm{T}}$ and $V_{\mathrm{T}}$ magnitudes using the relations $V=$ $V_{\mathrm{T}}-0.090\left(B_{\mathrm{T}}-V_{\mathrm{T}}\right)$ and $B-V=0.850\left(B_{\mathrm{T}}-V_{\mathrm{T}}\right)$, which are valid in the colour interval $-0.2 \mathrm{mag}<B_{\mathrm{T}}-V_{\mathrm{T}}<1.8 \mathrm{mag}$ (ESA 1997). For $\sigma$ Ori A and B, I used the $B$ and $V$ magnitudes derived in Sect. 2.4, while for $\sigma$ Ori C, I used the photometry from Greenstein \& Wallerstein (1958).

The $V$ vs. $B-V$ colour-magnitude diagram depicted in Fig. 4 illustrates the mass derivation from the models. In particular, I used the basic grid for solar metalicity $Z=0.02$ (Schaller et al. 1992) and conservative possible intervals in the age $\left(3_{-2}^{+7} \mathrm{Ma}\right)$ and in the heliocentric distance $\left(360_{-60}^{+70} \mathrm{pc}\right)$ of $\sigma$ Orionis. The most probable masses for each target and for each age-distance pair
Table 9. Masses of the young stars.

\begin{tabular}{|c|c|c|}
\hline Name & $\begin{array}{l}\text { Mass } \\
{\left[M_{\odot}\right]}\end{array}$ & Remarks $^{a}$ \\
\hline$\sigma$ Ori A & $18 \pm 6$ & $\overline{X X},(\mathrm{mIR})$ \\
\hline$\sigma$ Ori B & $12 \pm 3$ & XX \\
\hline$\sigma$ Ori $\mathrm{E}$ & $7.4_{-1.4}^{+1.5}$ & He rich, $\mathrm{XX}$ \\
\hline$\sigma$ Ori $\mathrm{D}$ & $6.8_{-1.2}^{+1.8}$ & \\
\hline HD 37699 & $4.4_{-0.7}^{-0.8}$ & mIR, XX \\
\hline HD 294271 & $3.9_{-0.6}^{+0.8}$ & \\
\hline HD $37525 \mathrm{AB}$ & $3.6_{-0.6}^{-0.0}$ & binary, XX \\
\hline HD 294272 A & $3.2 \pm 0.5$ & \\
\hline HD 294272 B & $3.0 \pm 0.5$ & \\
\hline HD 37333 & $3.0_{-0.4}^{+0.6}$ & \\
\hline HD 37564 & $3.1_{-0.4}^{+0.6}$ & \\
\hline$\sigma$ Ori C & $2.7 \pm 0.4$ & \\
\hline V1147 Ori & $3.5_{-0.3}^{+0.4}$ & XX \\
\hline HD 37686 & $2.3_{-0.1}^{+0.4}$ & \\
\hline HD 37545 & $2.2_{-0.1}^{+0.3}$ & \\
\hline HD 294275 & $2.1_{-0.1}^{+0.3}$ & \\
\hline HD 294297 & $1.7_{-0.1}^{+0.1}$ & $\mu$ \\
\hline HD 294268 & $1.6_{-0.1}^{+0.2}$ & $\mathrm{mIR}$ \\
\hline RX J0539.6-0242 AB & $1.8 \pm 0.1$ & SB, XX \\
\hline HD 294279 & $1.6 \pm 0.1$ & \\
\hline HD 294273 & $1.5 \pm 0.1$ & \\
\hline StHA 50 & $1.4 \pm 0.1$ & \\
\hline HD 294301 & $1.5 \pm 0.1$ & prob. \\
\hline GSC 04771-00962 & $1.4 \pm 0.1$ & prob. \\
\hline HD 294298 & $1.5 \pm 0.1$ & XX \\
\hline $4771-0950$ & $1.3 \pm 0.1$ & \\
\hline 2E 0535.4-0241 & $1.5 \pm 0.1$ & $\mathrm{XX}$ \\
\hline TYC 477012611 & $1.3 \pm 0.1$ & prob. \\
\hline TYC 477011291 & $1.3 \pm 0.1$ & prob. \\
\hline SO120532 & $1.2 \pm 0.1$ & \\
\hline
\end{tabular}

${ }^{a}$ Remarks: "mIR": IRAS source; "XX": moderate or strong X-ray emitter; "prob.": probable association member star without detected youth features. The rest are described in the text.

was computed from the mass- $M_{V}$ relationship given by the models. The masses with their uncertainties for each target are shown in Table 9. The edge of the conservative error bars correspond to the extreme cases of a younger, closer cluster ( $1 \mathrm{Ma}, 300 \mathrm{pc})$ and for an older, farther cluster (10 Ma, $430 \mathrm{pc})$, which in contrast are close to being ruled out by the isochronal matches to lowmass stars. The extended grid with high mass loss for massive stars (Meynet et al. 1994) provided identical results, except for $\sigma$ Ori A whose derived mass slightly varied within the uncertainties of the age and heliocentric distance of the cluster. The masses of HD $37525 \mathrm{AB}$ and RX J0539.6-0242 AB were not corrected for binarity.

There are three bright stars $(V<9.5 \mathrm{mag})$ that are $0.1-0.2$ mag redder than the isochrones and the sequence defined by the other cluster members in the colour-magnitude diagram. They are $\sigma$ Ori B (whose atmosphere could be suffering from the effects of the $09.5 \mathrm{~V}$ primary at only $\sim 90 \mathrm{AU}$ ), $\sigma$ Ori $\mathrm{E}$ (a helium rich star - i.e. the used models may be not applicable) and HD 37564 (which may have a disc, see Sect. 3.2), meaning that their derived masses must be treated cautiously. The rest of the bright stars also lie slightly to the red of the isochrones in the colour-magnitude diagram in Fig. 4. Since there is almost no sensitivity to the age of the cluster in the high-mass domain of the colour-magnitude diagram in Fig. 4, this redshift favours the nearest possible heliocentric distances to $\sigma$ Orionis. It thus implies that the cluster could be older than usually assumed. This 


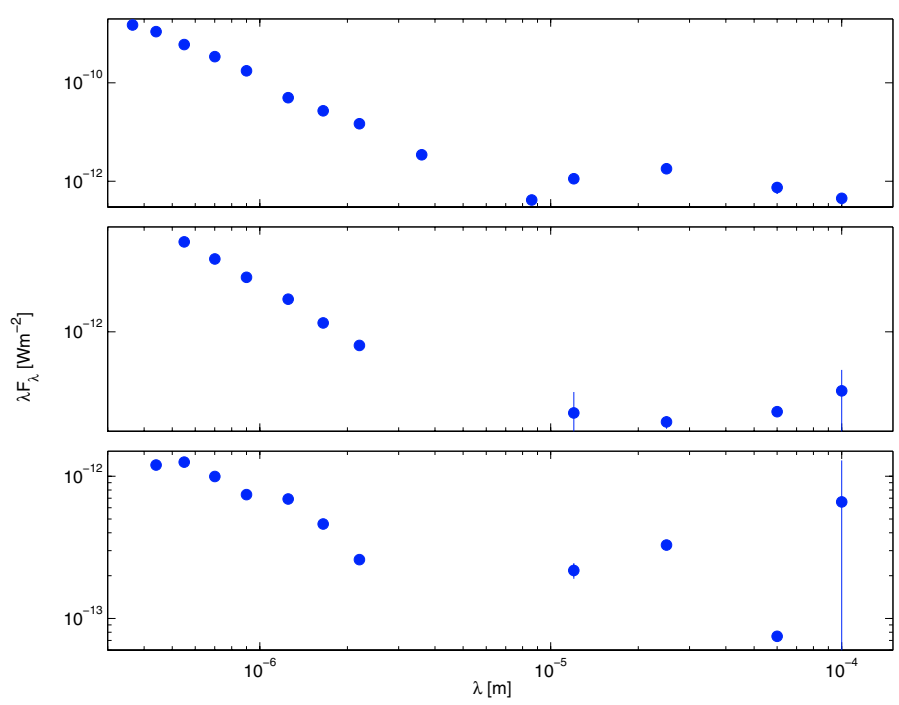

Fig. 5. Spectral energy distributions of $\sigma$ Ori A+B+IRS1 (top), HD 37699 (middle), and HD 294268 (bottom). Vertical scales are different.

fact is very important for the derivation of the mass function in the cluster substellar domain down to planetary masses (Béjar et al. 2001; González-García et al. 2006; Caballero et al. 2007a), since the theoretical masses from the models strongly depend on the age. An age older than expected for $\sigma$ Orionis, at about $10 \mathrm{Ma}$, would lead to dozens currently considered brown dwarfs being very low-mass stars, and to several planetary-mass objects being really brown dwarfs.

\subsection{Disc harbours and $X$-ray emitters}

Some of the young stars in Table 9 display characteristics that are present in $\mathrm{T}$ Tauri and Herbig Ae/Be stars. In the standard scenario, these stars possess discs, which are responsible for the flux excess in the mid and/or near infrared. The in-falling material from the disc onto the stellar surface produces the X-ray emission and the strong broad $\mathrm{H} \alpha$ emission. The columns of the accreting mass hitting the atmosphere can produce photometric variability and flux excess in the blue optical as well (Bertout 1989; Appenzeller \& Mundt 1989; Königl 1991; Hillenbrand et al. 1992).

Among the investigated stars, three were detected by the IRAS space telescope (Table 2 in Sect. 2.2.1). I built their spectral energy distributions (SEDs) from the blue optical to $100 \mu \mathrm{m}$ $\left((U) B V R I J H K_{\mathrm{s}}\left(L^{\prime}[8.6]\right)[12][25][60][100]\right)$, using the data presented here and from Lee (1968) and van Loon \& Oliveira (2003). There is high-resolution (sub-arcsec) imaging for only the $\sigma$ Ori $\mathrm{A}+\mathrm{B}+\mathrm{IRS} 1$ system. The mid-infrared source $\sigma$ Ori IRS1, at 3.3 arcsec northeast of the OB system $\sigma$ Ori AB, was resolved at 6-20 $\mu \mathrm{m}$ (van Loon \& Oliveira 2003), in X-rays (Sanz-Forcada et al. 2004), in the $J$ and $H$ bands (Caballero 2005), and in the optical (Rebolo et al. in prep.). It is responsible for the IRAS emission coming from the triple system. I propose that $\sigma$ Ori IRS1 is a low-mass K:-type star surrounded by a thick envelope that loses its outer atmospheric layers due to the strong ultraviolet radiation field and stellar wind coming from the $09.5 \mathrm{~V}+\mathrm{B} 0.5 \mathrm{~V}$ system at only $\sim 1000 \mathrm{AU}$ (see also van Loon \& Oliveira 2003). The two other stars with IRAS emission, HD 37699 and HD 294268, have masses of $4.4_{-0.7}^{+0.8}$ and $1.6_{-0.1}^{+0.2} M_{\odot}$, respectively, which makes them suitable Herbig Ae/Be star candidates. Besides, according to

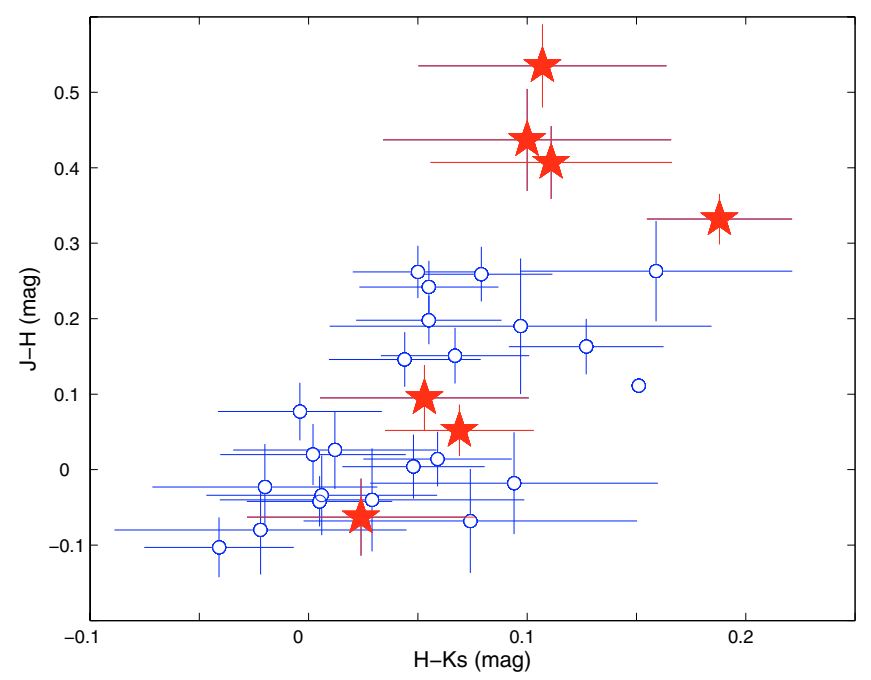

Fig. 6. $J-H$ vs. $H-K_{\mathrm{s}}$ colour-colour diagram of the brightest stars of the $\sigma$ Orionis cluster. Stars with and without disc are marked with filled stars and open circles, respectively. The error bars of $\sigma$ Ori AB have not been drawn for the sake of clarity.

Table 10. Young stars with probable discs ${ }^{a}$.

\begin{tabular}{lcr}
\hline \hline Name & Sp. type & Remarks \\
\hline HD 37699 & B5Vn & IRAS source \\
HD 37564 & A8V: & red $B-V$ \\
HD 294268 & F5 & IRAS source \\
RX J0539.6-0242 AB ${ }^{b}$ & G5-K0 & $V_{\mathrm{T}}-K_{\mathrm{s}}>2.0 \mathrm{mag}$ \\
StHA 50 & Be & blue $B-V$ \\
HD 294298 & G0: & $V_{\mathrm{T}}-K_{\mathrm{s}}>2.0 \mathrm{mag}$ \\
2E 0535.4-0241 & & $V_{\mathrm{T}}-K_{\mathrm{s}}>2.0 \mathrm{mag}$ \\
\hline
\end{tabular}

${ }^{a}$ Discarding $\sigma$ Ori IRS1.

${ }^{b}$ Weak-line T Tauri binary (Alcalá et al. 1996).

Bertout (1989), the most massive T Tauri stars rotate significantly faster than less massive ones. The large $v \sin i$ derived from the "nebulous" spectral type of HD 37699 (Neubauer 1943) points out that this relation may be extrapolated to Ae/Be stars. The disc surrounding HD 37699 also seems to be cooler than those of $\sigma$ Ori IRS1 and HD 294268, since the flux density at $60 \mu \mathrm{m}$ is larger than at $25 \mu \mathrm{m}$, in contrast to what happens in the other two. The large uncertainties in the IRAS fluxes prevent deriving additional properties of the discs. The abrupt step in the SED of HD 294268 between the BVRI-band data (Lee 1968) and the 2MASS $J H K_{\mathrm{s}}$-band data (Cutri et al. 2003) suggests a secular large-amplitude photometric variability.

There are five additional candidate star systems with probable discs. They are shown, together with HD 37699 and HD 294268, in Table 10. Three of them are the strong X-ray emitters with colours $V_{\mathrm{T}}-K_{\mathrm{s}}>2.0 \mathrm{mag}$ (Sect. 2.3.3). The remaining probable disc harbours are HD 37564 and StHA 50. The former is an A8:-type star with moderate X-ray emission and relatively red $B-V$ and $V_{\mathrm{T}}-K_{\mathrm{S}}$ colours. StHa 50, for which there is only a few data available (it is a Be-type emission-line object with $V \approx 11.4$ mag - Stephenson 1986; Downes \& Keyes 1988), deviates at the level of $3 \sigma$ to the blue in the $V$ vs. $B-V$ colour-magnitude diagram in Fig. 4 with respect to the sequence defined by the other cluster members. The IRAC Spitzer Space Telescope data between 4.5 and $8.0 \mu \mathrm{m}$ supports the hypothesis of discs surrounding both HD 37564 and StHA 50 (Hernández et al. 2007; Caballero et al. in prep.). As a matter of fact, both 


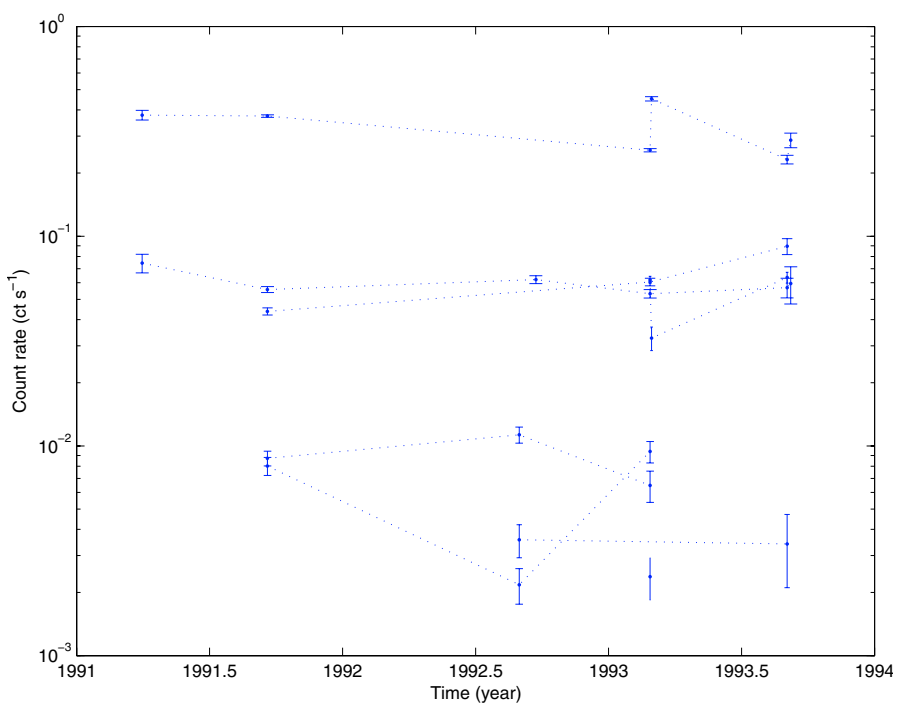

Fig. 7. X-ray light curves of the brightest $\sigma$ Orionis stars in the ROSAT WGACAT catalogue.

of them and HD 37699, the bluest star with a disc in $\sigma$ Orionis, have no appreciable near-infrared flux excess in the $J-H$ vs. $H-K_{\mathrm{s}}$ colour-colour diagram shown in Fig. 6. Their discs are probably cooler than those surrounding the remaining stars in Table 10.

The frequency of discs surrounding young stars in the magnitude interval $7.5 \mathrm{mag} \lesssim V \lesssim 11.0 \mathrm{mag}$ and in the direction of $\sigma$ Ori is $32 \pm 14 \%$. This value is of the order of other disc frequencies measured in the area in different mass intervals (compiled in Table 11). The frequencies derived from the detection of flux excess redwards of $5 \mu \mathrm{m}$ are in general larger than the disc frequencies based in only $J H K_{\mathrm{s}}$ photometry. Since the various disc frequencies quoted have been assessed heterogeneously with different techniques, I do not claim any evidence of a massdependence of the disc frequency in $\sigma$ Orionis.

The strongest $\mathrm{X}$-ray emitters are $\sigma$ Ori $\mathrm{A}+\mathrm{B}+\mathrm{IRS} 1$ and $\sigma$ Ori E. The $\sigma$ Ori $\mathrm{A}+\mathrm{B}+\mathrm{IRS} 1$ system was resolved in the $\mathrm{AB}$ and IRS1 components with HRI-C/Chandra Space Telescope observations. The bulk of the X-ray flux comes from the OB pair (Sanz-Forcada et al. 2004). Other moderate emitters are HD 37699, HD 37525 AB, V1147 Ori and the three stars with $V_{\mathrm{T}}-K_{\mathrm{s}}>2.0 \mathrm{mag}$ in Table 10 . Four bright stars out of the eight moderate and strong X-ray emitters seem to harbour surrounding discs, which supports the scenario of T Tauri and Ae/Be stars.

Figure 7 shows the variation between the years 1991 and 1994 in the background subtracted count rate in the energy interval $0.24-2.0 \mathrm{keV}$ of the flux of the young stars found in WGACAT. Several targets display flares and variability of the $\mathrm{X}$-ray emission on time scales of days to years. For example, the flux from $\sigma$ Ori $\mathrm{AB}$ (top light-curve in Fig. 7) raised from $0.257 \pm 0.005$ to $0.452 \pm 0.011 \mathrm{cts}^{-1}$ between 1993 Feb. 26 and 28, while the flux from V1147 Ori decreased a factor 5 with respect to a hypothetical quiescent level at $\sim 0.009 \mathrm{ct} \mathrm{s}^{-1}$ in observations spaced out about one year. Both $\sigma$ Ori AB and V1147 Ori do not apparently possess discs, so the X-ray variability is probably due to stellar winds and chromo- or magnetospheric effects, respectively.

\subsection{Different stellar populations surrounding $\sigma$ Ori}

There are five Hipparcos stars in the investigated area, whose HIP identifications and parallax determinations are provided in Table 12. The data may suggest that the stars are located at different heliocentric distances. However, the Hipparcos parallax measurements at more than $100 \mathrm{pc}$ are not quite reliable. Furthermore, in at least three cases the measurements are affected by multiplicity. It is expected that the GAIA mission will be able to disentangle distinct populations in the Ori OB1 association located at different heliocentric distances.

Meanwhile, these can be disentangle thanks to the knowledge of the spatial velocities of the young stars. Previous determinations of the radial velocity of $\sigma$ Ori AB and the mean radial velocity of the $\sigma$ Orionis cluster are found in a relatively narrow interval between +25 and $+35 \mathrm{~km} \mathrm{~s}^{-1}$ (Frost et al. 1926; Heard 1949; Wilson 1953; Evans 1967; Bolton 1974; Cruz-González et al. 1974; Conti et al. 1977; Morrell \& Levato 1991; Zapatero Osorio et al. 2002; Muzerolle et al. 2003; Burningham et al. 2005; Kenyon et al. 2005; Caballero 2005). The two recent studies by Jeffries et al. (2006) and Sacco et al. (2006) agree that the heliocentric radial velocity $\left(V_{r}\right)$ is about $+31.0 \pm 0.5 \mathrm{~km} \mathrm{~s}^{-1}$ with a dispersion less than $8 \mathrm{~km} \mathrm{~s}^{-1}$ (group 2 of Jeffries et al. 2006). I have compiled all the published values of $V_{r}$ of the investigated stars. They are provided in the last column of Table A.2. Among the nine stars with measured $V_{r}$, there is a non-member star (HD 294269, already discussed in Sects. 2.2.3 and 2.3.2). The remaining stars were classified in this work as members and possible members of the Ori OB $1 \mathrm{~b}$ association and $\sigma$ Ori $\mathrm{AB}, \mathrm{D}$, and $\mathrm{E}$ obviously display $V_{r}$ similar to the mean value of Jeffries et al. (2006). The same occurs for the classical T Tauri star RX J0539.6-0242 AB, at $\sim 15$ arcmin to the centre of the cluster. However, the measurements at two different epochs of the radial velocity of the $\mathrm{Ae} / \mathrm{Be}$ star HD 37699 differ from the mean radial velocity $\left(+14.8 \pm 2.2 \mathrm{~km} \mathrm{~s}^{-1}\right.$, Neubauer 1943; $+15 \mathrm{~km} \mathrm{~s}^{-1}$, Duflot et al. 1995). The radial velocity of HD 294298 is also quite discordant, $V_{r}=+11.5 \mathrm{~km} \mathrm{~s}^{-1}$. Both HD 37699 and HD 294298 are closely located (in the northeastern outer part of the studied area, in the vicinity of the Horsehead and Alnitak), have similar proper motions (identical within the error bars) and possess discs. HD $294297\left(V_{r}=+25 \mathrm{~km} \mathrm{~s}^{-1}\right)$ and HD 294268 $\left(V_{r}=+20 \mathrm{~km} \mathrm{~s}^{-1}\right)$ apparently display different $V_{r}$ from the mean value of $\sigma$ Orionis as well.

The eastern star in this sample, also close to the Horsehead Nebula, is HD 294297 (with discordant radial velocity and lithium in absorption; Sect. 2.5.1). Its proper motion is $\gtrsim 30$ mas a $^{-1}$ larger than that of $\sigma$ Ori AB and similar to those of probable foreground stars with large tangential velocities. The expected positions in the future of HD 294297 and of the other 29 young stars are shown in Fig. 8. While most of the stars keep their positions in the sky or float around, in the same period of time HD 294297 would have crossed the Horsehead Nebula and then be located out of the 30 arcmin-radius area.

From the existence of such young stars with peculiar spatial velocities, it can be derived that $(i)$ the astrometric criterion used in Sect. 2.3.2 to discard stars as possible cluster members could be too conservative (but necessary to minimize the number of contaminant foreground stars in the list of candidate young stars) and (ii) there are young stars in the line of sight of the $\sigma$ Orionis cluster that probably originated in a different star-forming region in the Ori OB $1 \mathrm{~b}$ association. The second item supports the hypothesis of the existence of at least two spatially superimposed components around $\sigma$ Ori exposed by Jeffries et al. (2006). They 
Table 11. Frequency of discs in different mass intervals in the $\sigma$ Orionis cluster.

\begin{tabular}{|c|c|c|c|}
\hline $\begin{array}{l}\text { Frequency } \\
(\%)\end{array}$ & $\begin{array}{l}\text { Mass interval } \\
\left(M_{\odot}\right)\end{array}$ & Method & Reference \\
\hline $17_{-17}^{+18}$ & $\sim 0.20-0.07$ & $L^{\prime}$ excess & Jawawardhana et al. (2003) \\
\hline$>5-12$ & $\sim 0.50-0.05$ & $K_{\mathrm{s}}$ excess & Barrado y Navascués et al. (2003) \\
\hline $46-54$ & $\sim 0.30-0.05$ & $K_{\mathrm{s}} L^{\prime}$ excess & Oliveira et al. (2004) \\
\hline$\sim 25$ & $\sim 0.07-0.05$ & $K_{\mathrm{s}} L^{\prime}$ excess, $\mathrm{H} \alpha$ emission, phot. variability & Caballero et al. (2004) \\
\hline $5-7$ & $\sim 0.20-0.05$ & $\mathrm{Ca}$ II triplet, $\mathrm{H} \alpha$ emission, phot. variability & Scholz \& Eislöffel (2004) \\
\hline $17.4 \pm 3.6^{a}$ & $\sim 5-2$ & $H K$ excess & Hernández et al. (2005) \\
\hline $10 \pm 5$ & $\sim 0.30-0.05$ & broad $\mathrm{H} \alpha$ emission & Kenyon et al. (2005) \\
\hline $46_{-13}^{+16}$ & $\sim 1.5-0.30$ & broad $\mathrm{H} \alpha$ emission & Caballero (2005) \\
\hline $33 \pm 6$ & $\sim 0.30-0.05$ & $K_{\mathrm{S}} L^{\prime}$ excess & Oliveira et al. (2006) \\
\hline $33 \pm 4$ & $\sim 1.0-0.035$ & $8.0 \mu \mathrm{m}$ excess & Caballero et al. (2006b) \\
\hline $47 \pm 14$ & $\sim 0.11-0.02$ & $5.8-8.0 \mu \mathrm{m}$ excess & Caballero et al. (2007a) \\
\hline$\sim 10-35$ & $\sim 5-0.07$ & $8.0-24.0 \mu \mathrm{m}$ excess & Hernández et al. (2007) \\
\hline$\sim 40$ & $\sim 0.012-0.008$ & $5.8 \mu \mathrm{m}$ excess & Caballero et al. (2007b) \\
\hline $32 \pm 14$ & $\sim 5-1.5$ & $K_{\mathrm{s}}$ excess, $12-100 \mu \mathrm{m}$ excess, abnormal $B-V$ & This work \\
\hline
\end{tabular}

${ }^{a}$ Frequency of discs around bright stars in the Ori OB 1 bc (super-)association using an $H K$ criterion.

Table 12. Young stars in the Hipparcos catalogue.

\begin{tabular}{lccr}
\hline \hline Name & HIP & $\begin{array}{c}\pi \pm \delta \pi \\
{[\mathrm{mas}]}\end{array}$ & $\begin{array}{r}\text { Sp. } \\
\text { type }\end{array}$ \\
\hline$\sigma$ Ori A+B+IRS1 & 26549 & $2.84 \pm 0.91$ & O9.5V+... \\
$\sigma$ Ori D & 26551 & $2.84 \pm 0.91$ & B2V \\
HD 37699 & 26694 & $0.63 \pm 1.01$ & B5Vn \\
HD 37525 AB & 26579 & $2.55 \pm 0.99$ & B5Vp \\
HD 37333 & 26438 & $2.52 \pm 1.08$ & A1Va \\
\hline
\end{tabular}

provided an excellent review of some possible consequences of a population with mixed age and distance. However, given that their spectroscopic study covered a large area, they investigated young stars that are out of the 30 arcmin-radius and closer to the extremely bright $\mathrm{O} 9.5 \mathrm{Ib}$-type Alnitak star than to $\sigma$ Ori AB itself. Three out of the proper-motion outlier young stars in this section are also located in the direction of Alnitak, very close to the Horsehead Nebula. What we see may be, in reality, an overlapping of sub-associations linked to different, but relatively close, star-forming regions. This was firstly noted by Béjar et al. (2004a), who proposed that an overdensity of photometric cluster members to the northeast of $\sigma$ Orionis could be associated to a hypothetical cluster surrounding Alnitak. They also studied the radial distribution of low-mass stars and brown dwarfs in a region around $\sigma$ Ori and found that the characteristic radius of the surface density, $\rho(r)$, was $r_{0}=8.8 \pm 0.6$ arcmin (where $\rho(r)=\rho_{0} \mathrm{e}^{-r / r_{0}}$ and $r$ is the separation to the cluster centre). Since the spatial distribution of stars (split in different mass intervals) and substellar objects in the cluster are identical (Caballero 2006), the spatial density of stellar members at $\sim 26$ arcmin $\left(r=3 r_{0}\right)$ is about $5 \%$ of the central density. Similar results were found by Sherry et al. (2004). Therefore, the contamination by young stars that do not belong to the $\sigma$ Orionis cluster in wide searches like this may be significant, especially at large separations to the centre. In particular, the four stars with abnormal spatial velocities are located at 21-28 arcmin to $\sigma$ Ori AB, which supports this hypothesis. The only way to make much headway against these problems is to obtain precise $V_{r}$ over a wider area, quantify the number of young contaminants and determine if they belong to any of the sub-groups presented by Jeffries et al. (2006) or to new ones. Multi-epoch observations are mandatory, since bona fide $\sigma$ Orionis member spectroscopic binaries could appear as $V_{r}$ outliers and vice versa. Besides, the

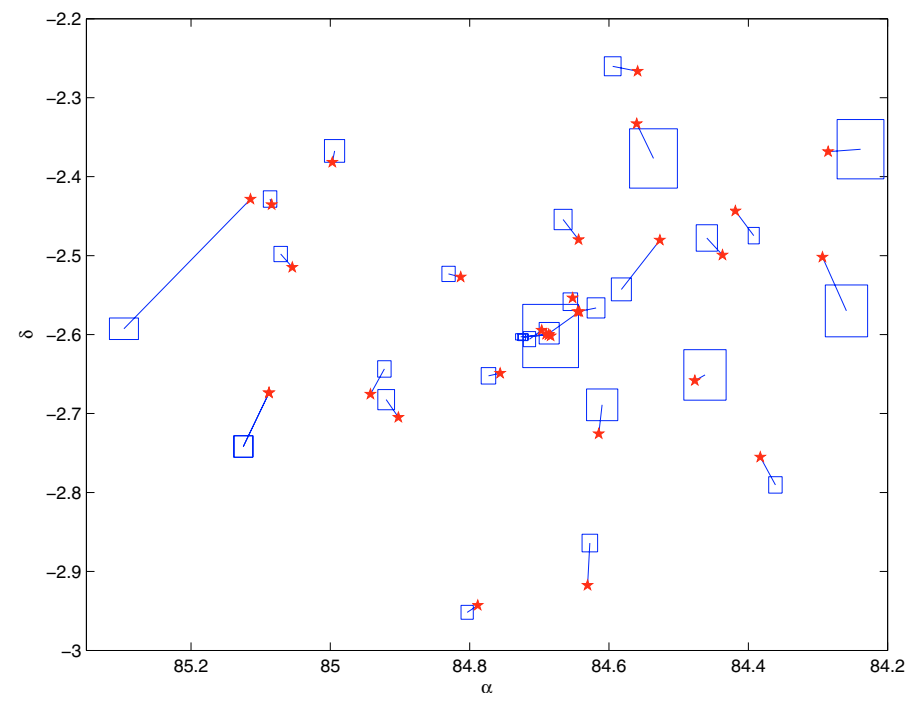

Fig. 8. Current (filled stars) and future (boxes) positions of the young stars and candidates. Sizes of the boxes are proportional to the uncertainties.

binary frequency in the higher mass domain of the cluster seems to be as high as $\sim 50 \%$ (Caballero 2005).

\subsection{Total mass and mass function from 1.1 to $24 M_{\odot}$}

The masses derived for the stars of this sample are about $20 \%$ less than previous determinations in the literature (e.g. Hunger et al. 1989 and Hernández et al. 2005). The list of the twelve stars in $\sigma$ Orionis that are more massive than $3 M_{\odot}$ basically matches that prepared by Sherry et al. (2004). They derived $\sim 100 M_{\odot}$ as the mass exclusively contained in them. However, in the present work, the stars with $M>3 M_{\odot}$ account for only $\sim 64 M_{\odot}$, which may lead to a cluster total mass significantly lower than previously estimated $\left(225 \pm 30 M_{\odot}\right.$; Sherry et al. 2004). Simply by scaling the correction factor down to lower masses, the cluster total mass could be at the level of only $\sim 150 M_{\odot}$. The total mass could even be lower accounting for probable young contaminants.

The total mass of the stars in this sample (probably all the $\sigma$ Orionis stars more massive than $\sim 1.2 M_{\odot}$ plus some young 


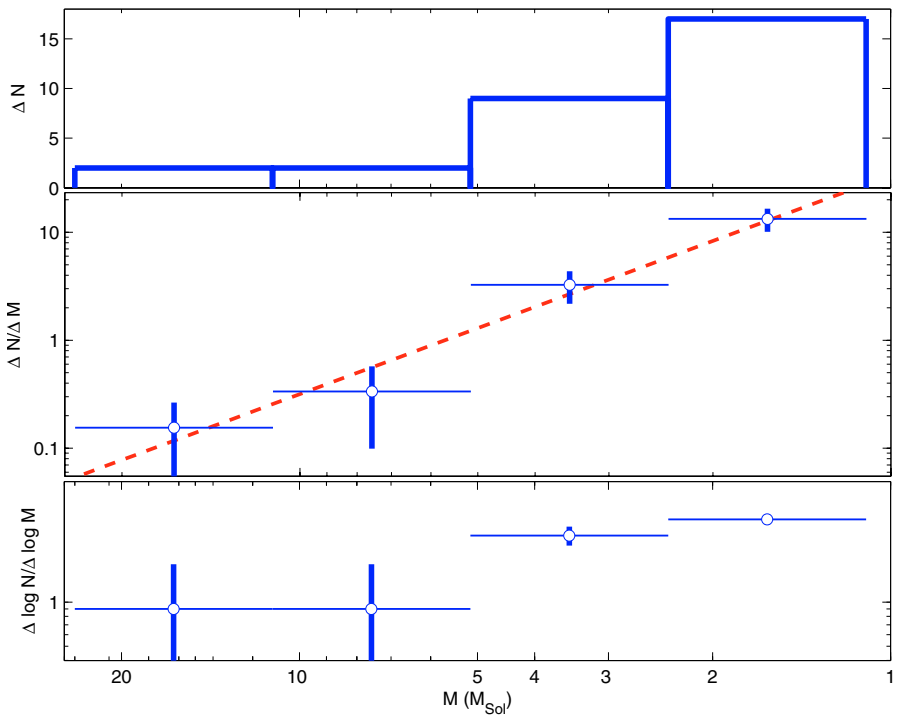

Fig. 9. The cumulative number of stars per mass interval (top), the mass spectrum (middle), and the mass function (bottom) in $\sigma$ Orionis in the mass interval 1.1-24 $M_{\odot}$. A distance of $360 \mathrm{pc}$ and an age of $3 \mathrm{Ma}$ have been used. In the middle window, the dashed (red) line marks the fit of the mass spectrum $(\alpha=+2.0)$.

contaminant) is $100_{-19}^{+20} M_{\odot}$. Out of it, $46 \%$ is contained in the $\sigma$ Ori $\mathrm{A}+\mathrm{B}+\mathrm{C}+\mathrm{D}+\mathrm{E}$ system, and up to $29 \%$ only in the two most massive stars in the cluster, $\sigma$ Ori A and B. This fact indicates that the cluster presents a well-defined cluster centre, in contrast to other extended star-forming regions, such as Upper Scorpius (de Zeeuw et al. 1999; Preibisch \& Zinnecker 1999). Discarding the three stars discussed in Sect. 3.3, the cluster barycentre is located at $\sim 1$ arcmin to the west of $\sigma$ Ori AB. The mean tangential velocity of the cluster centre of mass is $\left(\mu_{\alpha} \cos \delta, \mu_{\delta}\right)=$ $(+2.2 \pm 1.2,-0.5 \pm 1.0)$ mas a $^{-1}$, which is also quite similar to the tangential velocity of $\sigma$ Ori $\mathrm{AB}$ (the result after rejecting the three stars is identical within the error bars $)^{3}$.

Last, but not less important, I have also computed the mass spectrum in $\sigma$ Orionis in the mass interval 1.1-24 $M_{\odot}$ from the masses shown in Table 9. Figure 9 displays the cumulative number of stars per mass interval $(\Delta N)$, the mass spectrum $\left(\Delta N / \Delta N=a \mathrm{M}^{\gamma} ; \alpha \equiv-\gamma\right)$ and the mass function $\left(\Delta \log N / \Delta \log N=A \mathrm{M}^{\Gamma} ; \alpha \equiv 1-\Gamma\right)$ in the cluster. The value of the $\alpha$ index from the fit of the mass spectrum for $3 \mathrm{Ma}$ and $360 \mathrm{pc}$ gave $\alpha=+2.0$ (the fit rejecting the four stars in the Sect. 3.3 offers the same result). Considering the very conservative uncertainties in the age, and heliocentric distance, and repeating the fit for the less probable cases ( $1 \mathrm{Ma}$ and $300 \mathrm{pc} ; 10 \mathrm{Ma}$ and $430 \mathrm{pc}$ ), then the value of the index in the mass spectrum is $\alpha=+2.0_{-0.1}^{+0.2}$. This value is slightly lower than, but quite consistent with, the $\alpha$ value for massive and solar-like stars $(\alpha \approx+2.3$; Salpeter 1955). It may be due to the incompleteness of the search below $1.3 M_{\odot}$ $\left(V_{\mathrm{T}} \gtrsim 11.5 \mathrm{mag}\right)$. However, the measured $\alpha$ nicely extrapolates the Sherry et al. (2004) studies in $\sigma$ Orionis. They find that the cluster mass function in the mass interval $0.2-1.0 M_{\odot}$ is also consistent with that of the field stars in the same mass interval by Kroupa $(2001,2002)$. Therefore, the index of the mass spectrum in $\sigma$ Orionis keeps roughly constant at $\alpha \sim 2$ from 24 to $0.2 M_{\odot}$, where there is a turning point (first mentioned by

3 The standard deviation with respect to the mean proper motion (no considering HD 37699, HD 294298, and HD 294297) is $5.1 \mathrm{mas} \mathrm{a}^{-1}$, which transforms into a tangential velocity dispersion of $8.4 \mathrm{~km} \mathrm{~s}^{-1}$ at the heliocentric distance of $360 \mathrm{pc}$.
Caballero 2006). The $\alpha$ index below $0.2 M_{\odot}$ is established to be in the range +0.6-0.8 (Béjar et al. 2001; González-García et al. 2006). Tej et al. (2002), who measured the mass spectrum in an interval covering both sides of the turning point $\left(0.5-0.08 M_{\odot}\right)$, obtained an intermediate value of $\alpha=+1.2 \pm 0.1$. Now, the $\sigma$ Orionis cluster has become an excellent laboratory for studying the mass function from the most massive stars down to planetary-mass objects.

\section{Summary}

This work is devoted to identifying and characterising the brightest stars of the $\sigma$ Orionis cluster. The results presented here will complement searches of low-mass stars, brown dwarfs, and planetary-mass objects in the cluster.

From a correlation between the Tycho- 2 and 2MASS catalogues, and using additional information from X-ray and midinfrared space missions and from the literature, I have classified the brightest stars in a circular area of radius 30 arcmin centred in $\sigma$ Ori $\mathrm{AB}$ into $(i)$ members of the Ori OB $1 \mathrm{~b}$ association with youth features (26), (ii) candidate young stars (4), and (iii) probable foreground stars with red $V_{\mathrm{T}}-K_{\mathrm{s}}$ colours and/or large tangential velocities (16). Comparing the sequence of the young stars with the lowest photometric errors and evolutionary tracks in the $V$ vs. $B-V$ diagram suggests that $\sigma$ Orionis could be closer and older than previously thought.

I have derived the masses from theoretical $M_{V}$-mass relations for the young stars and candidate young stars and computed the first mass spectrum of the cluster between 1.1 and $24 M_{\odot}$. The $\alpha$ index from the fit of the mass spectrum is $+2.0_{-0.1}^{+0.2}$, which is very similar to the Salpeter's index. The cluster total mass could be as low as $\sim 150 M_{\odot}$.

From the flux excess in the mid and/or near infrared, the abnormal position in the $V$ vs. $B-V$ diagram and the strong or moderate X-ray emission, I have derived the existence of discs surrounding seven stars, four out of which are new. The frequency of discs in the magnitude interval $7.5 \mathrm{mag} \lesssim V \lesssim 11.0 \mathrm{mag}$ is $32 \pm 14 \%$.

Finally, the existence of some stars with abnormal tangential and radial velocities supports the hypothesis of overlapping stellar populations of different ages and kinematics in the direction of the $\sigma$ Orionis cluster.

\section{Appendix A: Stellar data}

Table A.1 provides the photometric data for the 41 stars in the Tycho-2/2MASS correlation (above the horizontal line) and the five missing stars in the Tycho-2/2MASS correlation (below the horizontal line), ordered by the $V_{\mathrm{T}}$-band magnitude. It is given the name of each star (if not available, then the Tycho identification is provided) and the $B_{\mathrm{T}^{-}}$and $V_{\mathrm{T}}$-band magnitudes from the Tycho-2 catalogue and the $J-, H$-, and $K_{\mathrm{s}}$-band magnitudes from the 2MASS catalogue.

Table A. 2 provides the astrometric data and the spectral types in the same manner as in Table A.1. It is given the name of each star, right ascension, and declination from the Tycho- 2 catalogue $($ FK5, Equinox $=$ J2000.0, Epoch $=$ J2000.000, proper motions taken into account), proper motions in right ascension and in declination from the Tycho-2 catalogue, and spectral types from the literature (references in the text).

Acknowledgements. I thank J. Adams, C. Bertout, D. J. Lennon, V. J. Sánchez Béjar, E. Solano, M. R. Zapatero Osorio, and especially, the referee, R. D. Jeffries, for helpful comments at different stages of the writing. Partial financial support was provided by the Spanish Ministerio de Ciencia y Tecnología project AYA2004-00253 of the Plan Nacional de Astronomía y Astrofísica. This 
Table A.1. Optical and near-infrared photometry of all the stars.

\begin{tabular}{|c|c|c|c|c|c|}
\hline Name & $\begin{array}{c}B_{\mathrm{T}} \pm \delta B_{\mathrm{T}} \\
{[\mathrm{mag}]}\end{array}$ & $\begin{array}{c}V_{\mathrm{T}} \pm \delta V_{\mathrm{T}} \\
{[\mathrm{mag}]}\end{array}$ & $\begin{array}{l}J \pm \delta J \\
{[\mathrm{mag}]}\end{array}$ & $\begin{array}{c}H \pm \delta H \\
{[\mathrm{mag}]}\end{array}$ & $\begin{array}{c}K_{\mathrm{s}} \pm \delta K_{\mathrm{s}} \\
{[\mathrm{mag}]}\end{array}$ \\
\hline$\sigma$ Ori $\mathrm{A}+\mathrm{B}+\mathrm{IRS} 1$ & 3.5210 .014 & 3.7630 .009 & 4.7520 .260 & 4.6410 .252 & 4.4900 .016 \\
\hline$\sigma$ Ori E & 6.2360 .014 & 6.3440 .010 & 6.9740 .026 & 6.9540 .031 & 6.9520 .029 \\
\hline HD 37699 & 7.4570 .015 & 7.5900 .011 & 7.8410 .029 & 7.9040 .042 & 7.8800 .031 \\
\hline HD 294271 & 7.7570 .016 & 7.8560 .011 & 8.1000 .021 & 8.1800 .055 & 8.2020 .038 \\
\hline HD $37525 \mathrm{AB}$ & 7.9520 .016 & 8.0580 .012 & 8.1310 .030 & 8.1050 .042 & 8.0930 .020 \\
\hline HD 294272 A & 8.4020 .017 & 8.3890 .015 & 8.3460 .026 & 8.3800 .046 & 8.3740 .026 \\
\hline HD $294272 \mathrm{~B}^{a}$ & 8.5440 .018 & 8.5540 .015 & 8.7790 .021 & 8.8190 .065 & 8.7900 .025 \\
\hline HD 37333 & 8.6160 .017 & 8.5080 .013 & 8.4130 .023 & 8.4810 .065 & 8.4070 .040 \\
\hline HD 37564 & 8.6990 .017 & 8.4430 .014 & 7.9760 .021 & 7.8810 .038 & 7.8280 .029 \\
\hline V1147 Ori & 9.0650 .018 & 8.9920 .016 & 8.8760 .024 & 8.8940 .063 & 8.8000 .019 \\
\hline HD 37686 & 9.2100 .019 & 9.1860 .018 & 9.2070 .035 & 9.2030 .024 & 9.1550 .022 \\
\hline HD 37545 & 9.2630 .018 & 9.2770 .017 & 9.2610 .023 & 9.3030 .024 & 9.2980 .023 \\
\hline HD 294275 & 9.5040 .020 & 9.4310 .019 & 9.2560 .028 & 9.2420 .023 & 9.1830 .025 \\
\hline HD 294268 & 10.9660 .049 & 10.5070 .043 & 9.3930 .023 & 9.0610 .024 & 8.8730 .023 \\
\hline HD 294307 & 11.0910 .054 & 10.5520 .046 & 9.4390 .026 & 9.1770 .023 & 9.1270 .019 \\
\hline $\mathrm{RX} \mathrm{J} 0539.6-0242 \mathrm{AB}^{b}$ & 11.1030 .055 & 10.1950 .036 & 8.4620 .027 & 8.0550 .040 & 7.9440 .038 \\
\hline HD 294279 & 11.1310 .054 & 10.6850 .053 & 9.8730 .028 & 9.7270 .023 & 9.6830 .026 \\
\hline EM* StHA 50 & 11.2190 .059 & 11.2750 .089 & 10.6660 .024 & 10.6140 .024 & 10.5450 .024 \\
\hline GSC 04771-00621 & 11.2310 .059 & 10.9200 .063 & 9.7960 .027 & 9.6000 .023 & 9.5030 .027 \\
\hline HD 294277 & 11.4160 .063 & 9.7370 .024 & 6.7730 .017 & 6.0660 .034 & 5.8610 .021 \\
\hline HD 294278 & 11.4490 .067 & 9.9410 .029 & 7.5920 .023 & 7.0340 .053 & 6.8550 .033 \\
\hline HD 294270 & 11.5070 .067 & 10.9600 .062 & 9.7800 .024 & 9.5110 .025 & 9.4720 .025 \\
\hline HD 294301 & 11.5410 .079 & 11.1390 .084 & 10.2100 .028 & 10.0590 .024 & 9.9920 .024 \\
\hline HD 294269 & 11.5970 .076 & 10.7950 .011 & 9.1780 .035 & 8.7210 .033 & 8.6340 .027 \\
\hline GSC 04771-00962 & 11.6080 .073 & 11.1010 .075 & 10.2020 .026 & 9.9600 .023 & 9.9050 .022 \\
\hline HD 294298 & 11.6460 .086 & 10.9750 .073 & 9.3390 .029 & 8.9020 .061 & 8.8020 .025 \\
\hline HD 294274 & 11.6620 .071 & 10.7580 .056 & 9.4280 .027 & 9.0690 .023 & 8.9850 .025 \\
\hline [W96] 4771-0950 & 11.8070 .086 & 11.4290 .099 & 10.0880 .027 & 9.8290 .024 & 9.7500 .022 \\
\hline HD 294280 & 11.8930 .091 & 9.8650 .027 & 6.4290 .019 & 5.5800 .031 & 5.3400 .024 \\
\hline TYC 47717201 & 11.9440 .105 & 11.7870 .152 & 10.2690 .028 & 9.8850 .023 & 9.8130 .021 \\
\hline 2E 0535.4-0241 & 12.0210 .117 & 11.0150 .069 & 9.2550 .020 & 8.7200 .051 & 8.6130 .025 \\
\hline TYC 477012611 & 12.2320 .160 & 11.4980 .106 & 10.7210 .048 & 10.4580 .046 & 10.2990 .042 \\
\hline TYC 47716611 & 12.3860 .201 & 11.8990 .178 & 10.6170 .026 & 10.2980 .026 & 10.2250 .024 \\
\hline TYC 477014321 & 12.7990 .255 & 12.3370 .211 & 11.0770 .023 & 10.8440 .025 & 10.7310 .023 \\
\hline TYC 477011291 & 12.8660 .240 & 11.8270 .146 & 11.2320 .021 & 11.0340 .024 & 10.9790 .023 \\
\hline TYC 477010181 & 13.0140 .280 & 11.2240 .077 & 8.6200 .021 & 7.8810 .027 & 7.7300 .017 \\
\hline SO120532 & 13.0940 .317 & 12.0310 .172 & 11.1250 .026 & 10.9620 .026 & 10.8350 .024 \\
\hline TYC 477110121 & 13.0090 .302 & 11.2630 .090 & 8.9980 .019 & 8.3650 .061 & 8.2050 .026 \\
\hline TYC 47719341 & 13.3250 .387 & 12.8700 .306 & 8.9480 .032 & 8.3740 .057 & 8.2070 .026 \\
\hline TYC 477114681 & 13.4330 .343 & 11.1360 .074 & 8.6160 .026 & 7.8930 .053 & 7.7270 .023 \\
\hline TYC 47709241 & 13.3670 .358 & 11.9380 .163 & 10.7250 .022 & 10.3170 .027 & 10.2220 .025 \\
\hline$\sigma$ Ori $\mathrm{D}$ & 6.3750 .012 & 6.5570 .014 & 7.1160 .029 & 7.2190 .027 & 7.2600 .021 \\
\hline$\sigma$ Ori $\mathrm{C}^{e}$ & 8.770 .02 & 8.790 .02 & 9.0860 .032 & 9.1090 .047 & 9.1290 .021 \\
\hline HD 294273 & 10.8990 .106 & 10.8280 .186 & 10.1760 .028 & 10.0990 .026 & 10.1030 .027 \\
\hline HD 294297 & 10.9050 .090 & 10.1930 .073 & 9.0620 .032 & 8.8720 .084 & 8.7750 .024 \\
\hline HD 294276 & 11.1930 .071 & 10.4180 .057 & 9.1830 .021 & 8.8560 .025 & 8.8140 .026 \\
\hline
\end{tabular}

\footnotetext{
${ }^{a}$ Also BD-02 1323C.

${ }^{b}$ Also 2E 0537.1-0243.

${ }^{c}$ Name from Wolk (1996).

Also SO210457.

${ }^{d}$ Name from Caballero (2006).

${ }^{e}$ Johnson $B$ - and $V$-band photometry from Greenstein \& Wallerstein (1958).
}

research made use of the SIMBAD database, operated at the CDS, Strasbourg, France. This publication makes use of data products from the Two Micron All Sky Survey, which is a joint project of the University of Massachusetts and the Infrared Processing and Analysis Center/California Institute of Technology, funded by the National Aeronautics and Space Administration and the National Science Foundation. 
Table A.2. Astrometry, spectral type, and radial velocity (when available) of all the stars.

\begin{tabular}{|c|c|c|c|c|c|c|c|}
\hline Name & TYC & $\begin{array}{c}\alpha \\
\text { (ICRS) }\end{array}$ & $\begin{array}{c}\delta \\
(\mathrm{ICRS})\end{array}$ & $\begin{array}{c}\mu_{\alpha} \cos \delta \\
\pm \delta \mu_{\alpha} \cos \delta \\
{\left[\mathrm{mas} \mathrm{a}^{-1}\right]}\end{array}$ & $\begin{array}{c}\mu_{\delta} \\
\pm \delta \mu_{\delta} \\
{\left[\mathrm{mas} \mathrm{a}^{-1}\right]}\end{array}$ & $\begin{array}{l}\text { Sp. } \\
\text { type }\end{array}$ & $\begin{array}{c}V_{r} \\
\mathrm{~km} \mathrm{~s}^{-1}\end{array}$ \\
\hline$\sigma$ Ori $\mathrm{A}+\mathrm{B}+\mathrm{IRS} 1^{a}$ & 477111961 & 053844.765 & -023600.25 & $+4.61 \pm 0.88$ & $-0.40 \pm 0.53$ & $09.5 \mathrm{~V}+\ldots$ & +29.1 \\
\hline$\sigma$ Ori E & 477111941 & 053847.208 & -023540.52 & $+2.2 \pm 1.1$ & $-1.4 \pm 1.2$ & B2Vp & +29 \\
\hline HD 37699 & 477110451 & 054020.189 & -022608.22 & $+0.3 \pm 1.2$ & $+0.9 \pm 1.3$ & B5Vn & $+14.8 \pm 2.2$ \\
\hline HD 294271 & 477111931 & 053836.549 & -023312.76 & $+0.4 \pm 1.3$ & $-0.6 \pm 1.4$ & B5V & \\
\hline HD $37525 \mathrm{AB}$ & 477111031 & 053901.493 & -023856.36 & $+2.1 \pm 1.3$ & $-0.4 \pm 1.3$ & B5Vp & \\
\hline HD 294272 A & 477112051 & 053834.799 & -023415.78 & $-3.3 \pm 1.6$ & $+0.6 \pm 1.6$ & B9.5III & \\
\hline HD 294272 B & 477112041 & 053834.235 & -023416.08 & $+5.4 \pm 1.8$ & $-3.4 \pm 1.7$ & B8V & \\
\hline HD 37333 & 47719561 & 053740.476 & -022636.83 & $-3.3 \pm 1.0$ & $-3.9 \pm 1.3$ & A1Va & \\
\hline HD 37564 & 477110731 & 053915.061 & -023137.62 & $+2.2 \pm 1.2$ & $+0.5 \pm 1.2$ & A8V: & \\
\hline V1147 Ori & 47716851 & 053946.196 & -024032.06 & $-2.5 \pm 1.2$ & $+4.0 \pm 1.3$ & B9IIIp & \\
\hline HD 37686 & 47719091 & 054013.090 & -023053.18 & $+2.1 \pm 1.2$ & $+2.1 \pm 1.2$ & B9.5Vn & \\
\hline HD 37545 & 47713041 & 053909.213 & -025634.73 & $+1.9 \pm 1.1$ & $-1.1 \pm 1.1$ & B9V & \\
\hline HD 294275 & 4771391 & 053731.872 & -024518.48 & $-2.7 \pm 1.2$ & $-4.4 \pm 1.3$ & A1V & \\
\hline HD 294268 & 47718021 & 053814.121 & -021559.78 & $+4.5 \pm 1.5$ & $+0.8 \pm 1.5$ & F5 & +20 \\
\hline HD 294307 & 47711571 & 054012.462 & -025257.59 & $+0.1 \pm 1.4$ & $-16.8 \pm 1.4$ & F8 & \\
\hline RX J0539.6-0242 AB & 47715431 & 053936.541 & -024217.21 & $+2.2 \pm 1.5$ & $+2.8 \pm 1.6$ & G5-K0 & $+32.6 \pm 1.8$ \\
\hline HD 294279 & 47714051 & 053831.382 & -025503.15 & $-0.4 \pm 1.4$ & $+6.7 \pm 1.4$ & F3-5 & \\
\hline StHA 50 & 477110871 & 053834.449 & -022847.56 & $+2.8 \pm 1.6$ & $+3.2 \pm 1.6$ & $\mathrm{Be}$ & \\
\hline GSC 04771-00621 & 47716211 & 053741.793 & -022908.21 & $+7.0 \pm 1.6$ & $-11.1 \pm 1.6$ & & \\
\hline HD 294277 & 47714631 & 053757.342 & -025317.69 & $+4.8 \pm 1.2$ & $-2.8 \pm 1.2$ & K2 & \\
\hline HD 294278 & 47711271 & 053838.762 & -024901.31 & $+10.9 \pm 1.4$ & $+2.6 \pm 1.4$ & K2 & \\
\hline HD 294270 & 477013361 & 053718.818 & -023136.44 & $+16.8 \pm 1.9$ & $-23.4 \pm 1.8$ & G0 & \\
\hline HD 294301 & 47715561 & 054021.125 & -024025.62 & $+4.6 \pm 1.7$ & $-8.5 \pm 1.7$ & $\mathrm{~F} 2 \mathrm{~V}(\mathrm{n})$ & \\
\hline HD 294269 & 47716931 & 053757.818 & -022633.65 & $+43.5 \pm 1.8$ & $-16.1 \pm 1.8$ & G0 & +72.8 \\
\hline GSC 04771-00962 & 47719621 & 053744.915 & -022957.31 & $+2.8 \pm 1.9$ & $+2.7 \pm 2.1$ & & \\
\hline HD 294298 & 47718801 & 053959.318 & -022254.35 & $-0.4 \pm 1.8$ & $+1.8 \pm 1.8$ & G0: & +11.5 \\
\hline HD 294274 & 47719401 & 053745.365 & -024412.53 & $-8.1 \pm 3.2$ & $-19.4 \pm 3.5$ & G0 & \\
\hline 4771-0950 & 47719501 & 053806.496 & -022849.37 & $+6.9 \pm 1.8$ & $-7.8 \pm 1.8$ & F7 & \\
\hline HD 294280 & 47713851 & 053828.491 & -030333.79 & $-4.0 \pm 1.7$ & $+4.9 \pm 1.8$ & K5 & \\
\hline TYC 47717201 & 47717201 & 053759.046 & -024100.47 & $-20.1 \pm 3.9$ & $-16.6 \pm 4.2$ & & \\
\hline 2E 0535.4-0241 & 47719211 & 053754.405 & -023929.84 & $-1.8 \pm 3.8$ & $+0.9 \pm 4.0$ & & \\
\hline TYC 477012611 & 477012611 & 053710.470 & -023007.19 & $-4.3 \pm 3.8$ & $-8.5 \pm 4.1$ & & \\
\hline TYC 47716611 & 47716611 & 053920.446 & -022751.44 & $+16.6 \pm 4.3$ & $-20.1 \pm 4.7$ & & \\
\hline TYC 477014321 & 477014321 & 053709.689 & -023959.87 & $-30.9 \pm 4.5$ & $+0.1 \pm 5.0$ & & \\
\hline TYC 477011291 & 477011291 & 053708.495 & -022206.34 & $-5.8 \pm 4.2$ & $+0.4 \pm 4.7$ & & \\
\hline TYC 477010181 & 477010181 & 053657.142 & -022540.00 & $-4.3 \pm 4.2$ & $-2.3 \pm 4.6$ & & \\
\hline SO120532 & 47718731 & 053814.438 & -021958.64 & $-3.0 \pm 4.3$ & $-5.5 \pm 4.7$ & F7-9 & \\
\hline TYC 477110121 & 477110121 & 053913.944 & -021049.27 & $-0.9 \pm 4.2$ & $-9.7 \pm 4.6$ & & \\
\hline TYC 47719341 & 47719341 & 053943.058 & -022845.59 & $-7.5 \pm 4.6$ & $-2.7 \pm 4.9$ & & \\
\hline TYC 477114681 & 477114681 & 053729.879 & -024345.83 & $-7.0 \pm 4.7$ & $-6.5 \pm 4.9$ & & \\
\hline TYC 47709241 & 47709241 & 053719.240 & -022846.22 & $+1.8 \pm 4.7$ & $-43.4 \pm 5.1$ & & \\
\hline$\sigma$ Ori $\mathrm{D}^{b}$ & 477111951 & 053845.602 & -023558.84 & $+4.6 \pm 0.9$ & $-0.4 \pm 0.5$ & $\mathrm{~B} 2 \mathrm{~V}$ & $+33.1 \pm 3.1$ \\
\hline$\sigma$ Ori $\mathrm{C}^{c}$ & - & 053844.12 & -023606.3 & $0 \pm 0$ & $0 \pm 0$ & $\mathrm{~A} 2 \mathrm{~V}$ & \\
\hline HD $294273^{b}$ & 477110231 & 053827.524 & -024332.60 & $-0.6 \pm 2.8$ & $+4.6 \pm 2.5$ & A3 & \\
\hline HD $294297^{b}$ & 477110811 & 054027.531 & -022542.90 & $+22.7 \pm 2.6$ & $-20.5 \pm 1.7$ & F6-8 & +25 \\
\hline HD $294276^{b}$ & 477014891 & 053720.671 & -024932.55 & $+8.8 \pm 1.7$ & $-61.7 \pm 3.6$ & G0 & \\
\hline
\end{tabular}

${ }^{a}$ Proper motions from Perryman et al. (1997). The spectral types of the components of the triple system are O9.5V, B0.5V and K:

${ }^{b}$ Proper motions from Høg et al. (1998).

${ }^{c}$ Coordinates from the 2MASS catalogue and proper motion from the NOMAD1 catalogue.

\section{References}

Adams, N. R., Wolk, S. J., Walter, F. M., \& Sherry, W. H. 2004, Am. Astron. Soc. Meet. 205, \#105.08

Alcalá, J. M., Terranegra, L., Wichmann, R., et al. 1996, A\&AS, 119, 7

Alcalá, J. M., Covino, E., Torres, G., et al. 2000, A\&A, 353, 186

Appenzeller, I., \& Mundt, R. 1989, A\&ARv, 1, 291

Barrado y Navascués, D., Zapatero Osorio, M. R., Béjar, V. J. S., et al. 2001, A\&A, 377, L9

Barrado y Navascués, D., Béjar, V. J. S., Mundt, R., et al. 2003, A\&A, 404, 171 Béjar, V. J. S., Zapatero Osorio, M. R., \& Rebolo, R. 1999, ApJ, 521, 671

Béjar, V. J. S., Martín, E. L., Zapatero Osorio, M. R., et al. 2001, ApJ, 556, 830

Béjar, V. J. S., Caballero, J. A., Rebolo, R., Zapatero Osorio, M. R., \& Barrado y Navascués, D. 2004a, Ap\&SS, 292, 339
Béjar, V. J. S., Zapatero Osorio, M. R., \& Rebolo, R. 2004b, AN, 325, 705 Bertout, C. 1989, ARA\&A, 27, 351

Blaauw, A. 1964, ARA\&A, 2, 213

Blaauw, A. 1991, The Physics of Star Formation and Early Stellar Evolution, NATO Advanced Science Institutes (ASI) Series C, Vol. 342, held in Agia Pelagia, Crete, Greece, May 27th-June 8th, Dordrecht: Kluwer, 1991, ed. C. J. Lada, \& N. D. Kylafis, 125

Bolton, C. T. 1974, ApJ, 192, L7

Bonnarel, F., Fernique, P., Bienaymé, O., et al. 2000, A\&AS, 143, 33

Briceño, C., Calvet, N., Hernández, J., et al. 2005, AJ, 129, 907

Brown, A. G. A., de Geus, E. J., \& de Zeeuw, P. T. 1994, A\&A, 289, 101

Brown, A. G. A., Walter, F. M., \& Blaauw, A. 1999, in ASP Conf. Ser., The Orion Complex Revisited, ed. M. J. McCaughrean, \& A. Burkert, in press 
ten Brummelaar, T., Mason, B. D., McAlister, H. A., et al. 2000, AJ, 119, 2403 Burningham, B., Naylor, T., Littlefair, S. P., \& Jeffries, R. D. 2005, MNRAS, 356,1583

Caballero, J. A. 2005, Astron. Nachr., 326, No. 10, 1007

Caballero, J. A. 2006, Ph.D. Thesis, Universidad de La Laguna

Caballero, J. A., Béjar, V. J. S., Rebolo, R., \& Zapatero Osorio, M. R. 2004, A\&A, 424, 857

Caballero, J. A., Martín, E. L., Dobbie, P. D., \& Barrado y Navascués 2006a, A\&A, 460, 635

Caballero, J. A., Barrado y Navascués, Béjar, V. J. S., et al. 2006b, in The 14th Cambridge Workshop on Cool Stars, Stellar Systems and the Sun, 6-10 Nov. 2006, Pasadena, CA, poster \#39

Caballero, J. A., et al. 2007a, A\&A, submitted

Caballero, J. A., et al. 2007b, A\&A, submitted

Catalano, F. A., \& Renson, P. 1998, A\&AS, 127, 421

Conti, P. S., Leep, E. M., \& Lorre, J. J. 1977, ApJ, 214, 759

Cruz-González, C., Recillas-Cruz, E., Costero, R., Peimbert, M., \& Torres-Peimbeert, S. 1974, RMxAA, 1, 211

Cunha, K., Smith, V. V., \& Lambert, D. L. 1995, ApJ, 452, 634

Cutri, R. M., Skrutskie, M. F., van Dyk, S., et al. 2003, VizieR On-line Data Catalog: II/246. Originally published in University of Massachusetts and Infrared Processing and Analysis Center (IPAC/California Institute of Technology)

Downes, R. A., \& Keyes, C. D. E. 1988, AJ, 96, 777

Duflot, M., Figon, P., \& Meyssonnier, N. 1995, A\&AS, 114, 269

European Space Agency, The Hipparcos and Tycho catalogues, ESA SP-1200, 1997

Evans, D. S. 1967, Determination of Radial Velocities and their Applications, Proc. IAU Symp. 30, held at the University of Toronto 20-24 June, 1966, ed. A. H. Batten, \& J. F. Heard (London: Academic Press), 57

Fernique, P., Bonnarel, F., Louys, M., et al. 2004, Toward an International Virtua Observatory: Proceedings of the ESO/ESA/NASA/NSF Conference Held at Garching, Germany, 10-14 June 2002, ESO Astrophys. Symp., ed. P. J. Quinn, \& K. M. Górski (Berlin, Heidelberg: Springer-Verlag), 271

Franciosini, E., Pallavicini, R., \& Sanz-Forcada, J. 2006, A\&A, 446, 501

Frost, E. B., Barrett, S. B., \& Struve, O. 1926, ApJ, 64, 1

García-Lario, P., Manchado, A., Suso, S. R., Pottasch, S. R., \& Olling, R. 1990, A\&AS, 82, 497

García-Lario, P., Manchado, A., Pych, W., \& Pottasch, S. R. 1997, A\&AS, 126, 479

Garrison, R. F. 1967, PASP, 79, 433

Genzel, R., \& Stutzki, J. 1989, A\&A, 27, 41

de Geus, E. J., Lub, J., \& van de Grift, E. 1990, A\&AS, 85, 915

Gieseking, F. 1983, A\&A, 118, 102

González-García, B. M., Zapatero Osorio, M. R., Béjar, V. J. S., et al. 2006, A\&A, 460, 799

Gray, R. O., \& Corbally, C. J. 1993, AJ, 106, 632

Greenstein, J. L., \& Wallerstein, G. 1958, ApJ, 127, 237

Gregorio-Hetem, J., \& Hetem, A. Jr. 2000, Planetary Systems in the Universe, International Astronomical Union. Symp. 202, Manchester, England, August 2000, meeting abstract 79

Goudis, C. 1982, The Orion complex: A case study of interstellar matter (Dordrecht, Netherlands: D. Reidel Publishing Co.), Astrophysics and Space Science Library, Vol. 90

Guetter, H. H. 1981, AJ, 86, 1057

Haro, G., \& Moreno, A. 1953, BOTT, 1g, 11

Hardie, R. H., Heiser, A. M., \& Tolbert, C. R. 1964, ApJ, 140, 1472

Heard, J. F. 1949, ApJ, 109, 185

Hernández, J., Calvet, N., Hartmann, L., Briceño, C., Sicilia-Aguilar, A., \& Berlind, P. 2005, AJ, 129, 856

Hernández, J., Hartmann, L., Megeath, T., et al. 2007, ApJ, submitted [arXiv:astro-ph/0701476]

Hillenbrand, L. A., Strom, S. E., Vrba, F. J., \& Keene, J. 1992, ApJ, 397, 613

Høg, E., Kuzmin, A., Bastian, U., et al. 1998, A\&A, 335, L65

Høg, E., Fabricius, C., Makarov, V. V., et al. 2000, A\&A, 355, L27

Hunger, K., Heber, U., \& Groote, D. 1989, A\&A, 224, 57

Jayawardhana, R., Ardila, D. R., \& Stelzer, B. 2003, Brown Dwarfs, Proceedings of IAU Symposium \#211, held 20-24 May 2002 at University of Hawai'i, Honolulu, Hawai'i, ed. E. L. Martín (San Francisco: ASP), 139

Jeffries, R. D., Maxted, P. F. L., Oliveira, J. M., \& Naylor, T. 2006, MNRAS, 371, L6

Kenyon, M. J., Jeffries, R. D., Naylor, T., Oliveira, J. M., \& Maxted, P. F. L. 2005, MNRAS, 356, 89

Kharchenko, N. V., Piskunov, A. E., Röser, S., Schilbach, E., \& Scholz, R.-D. 2005, A\&A, 440, 403
Kogure, T., Yoshida, S., Wiramihardja, S. D., et al. 1989, PASJ, 41, 1195

Königl, A. 1991, ApJ, 370, L39

Kraemer, K. E., Shipman, R. F., Price, S. D., et al. 2003, AJ, 126, 1423

Kroupa, P. 2001, MNRAS, 322, 231

Kroupa, P. 2002, Science, 295, 82

Lee, T. A. 1968 , ApJ, 152, 913

Lejeune, T., \& Schaerer, D. 2001, A\&A, 366, 538

van Loon, J. Th., \& Oliveira, J. M. 2003, A\&A, 405, L33

Lyngå, G. 1981, The Catalogue of Open Star Clusters, ADCBu, 1, 90

Martín, E. L., Zapatero Osorio, M. R., Barrado y et al. 2001, ApJ, 558, L117

Mayne, N. J., Naylor, T., Littlefair, S. P., Saunders, E. S., \& Jeffries, R. D. 2006, MNRAS, 375, 1220

Meynet, G., Maeder, A., Schaller, G., Schaerer, D., \& Charbonnel, C. 1994, A\&AS, 103, 97

Monet, D. G., Levine, S. E., Casian, B., et al. 2003, AJ, 125, 984

Morrell, N., \& Levato, H. 1991, ApJS, 75, 965

Muzerolle, J., Hillenbrand, L., Calvet, N., Briceño, C., \& Hartmann, L. 2003, ApJ, 592, 266

Nakano, M., Wiramihardja, S. D., \& Kogure, T. 1995, PASJ, 47, 889

Nesterov, V. V., Kuzmin, A. V., Ashimbaeva, N. T., et al. 1995, A\&AS, 110, 367

Neubauer, F. J. 1943, ApJ, 97, 300

Nissen, P. E. 1976, A\&A, 50, 343

Oganesyan, R. Kh., Epremyan, R. A., \& Karapetyan, A. A. 1995, Ap, 38, 189

Oliveira, J. M., \& van Loon, J. Th. 2004, A\&A, 418, 663

Oliveira, J. M., Jeffries, R. D., Kenyon, M. J., Thompson, S. A., \& Naylor, T. 2002, A\&A, 382, L22

Oliveira, J. M., Jeffries, J. D., \& van Loon, J. Th. 2004, MNRAS, 347, 1327

Oliveira, J. M., Jeffries, R. D., van Loon, J. Th., \& Rushton, M. T. 2006, MNRAS, 369, 272

Perryman, M. A. C., Lindegren, L., Kovalevsky, J., et al. 1997, A\&A, 323, L49

Preibisch, T., \& Zinnecker, H. 1999, AJ, 117, 2318

Reipurth, B., Bally, J., Fesen, R. A., \& Devine, D. 1998, Nature, 396, 343

ROSAT Scientific Team 2000a, ROSAT News, 71

ROSAT Scientific Team 2000b, ROSAT News, 72

Sacco, G. G., Randich, S., Franciosini, E., Pallavicini, R., \& Palla, F. 2006, A\&A, 462, L23

Salpeter, E. E. 1955, ApJ, 121, 161

Sanz-Forcada, J., Franciosini, E., \& Pallavicini, R. 2004, A\&A, 421, 715

Schaller, G., Schaerer, D., Meynet, G., \& Maeder, A. 1992, A\&AS, 96, 269

Schild, R. E., \& Chaffee, F. 1971, ApJ, 169, 529

Scholz, A., \& Eislöffel, J. 2004, A\&A, 419, 249

Sherry, W. H., Walter, F. M., \& Wolk, S. J. 2004, AJ, 128, 2316

Stephenson, C. B. 1986, AJ, 92, 139

Tej, A., Sahu, K. C., Chandrasekhar, T., \& Ashok, N. M. 2002, ApJ, 578, 523

Torres, C. A. O., Quast, G., de La Reza, R., Gregorio-Hetem, J., \& Lepine, J. R. D. 1995, AJ, 109, 2146

Voges, W., Aschenbach, B., Boller, Th., et al. 1999, A\&A, 349, 389

Walter, F. M., Wolk, S. J., Freyberg, M., \& Schmitt, J. H. M. M. 1997, MmSAI, 68,108

Walter, F. M., Wolk, S. J., \& Sherry, W. 1998, ASP Conf. Ser., 154, CD-1793

Warren, W. H. Jr., \& Hesser, J. E. 1977a, ApJS, 34, 115

Warren, W. H. Jr., \& Hesser, J. E. 1977b, ApJS, 34, 207

Warren, W. H. Jr., \& Hesser, J. E. 1978, ApJS, 36, 497

Weaver, Wm. B., \& Babcock, A. 2004, PASP, 116, 1035

White, N. E., Giommi, P., \& Angelini, L. 2001, VizieR On-line Data Catalog: IX/31. Originally published in: Laboratory for High Energy Astrophysics (LHEA/NASA), Greenbelt (2000)

Wilson, R. E. 1953, QB, 901, W495, General catalogue of stellar radial velocities. Washington, (Carnegie Institution of Washington), 1953

Wiramihardja, S. D., Kogure, T., Yoshida, S., Ogura, K., \& Nakano, M. 1989, PASJ, 41, 155

Wiramihardja, S. D., Kogure, T., Yoshida, S., et al. 1991, PASJ, 43, 27

Wolk, S. J. 1996, Ph.D. Thesis, State Univ. New York at Stony Brook

Wolk, S. J., \& Walter, F. M. 2000, Very Low-mass Stars and Brown Dwarfs, ed. R. Rebolo, \& M. R. Zapatero Osorio, 38

Zacharias, N., Monet, D. G., Levine, S. E., et al. 2004, Am. Astron. Soc. Meet., 205, \#48.15; BAAS, 36, 1418

Zapatero Osorio, M. R., Béjar, V. J. S., Martín, E. L., et al. 2000, Science, 290, 103

Zapatero Osorio, M. R., Béjar, V. J. S., Pavlenko, Ya., et al. 2002, A\&A, 384, 937

de Zeeuw, P. T., Hoogerwerf, R., de Bruijne, J. H. J., Brown, A. G. A., \& Blaauw, A. 1999, AJ, 117,354 